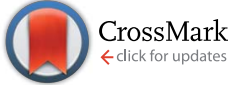

Cite this: J. Mater. Chem. A, 2016, 4, 3100

Received 13th September 2015 Accepted 16th December 2015

DOI: $10.1039 / c 5 t a 07328 f$

www.rsc.org/MaterialsA

\section{Utilizing modeling, experiments, and statistics for the analysis of water-splitting photoelectrodes $\uparrow$}

\begin{abstract}
Yannick K. Gaudy and Sophia Haussener*
A multi-physics model of a planar water-splitting photoelectrode was developed, validated, and used to identify and quantify the most significant materials-related bottlenecks in photoelectrochemical device performance. The model accounted for electromagnetic wave propagation within the electrolyte and semiconductor, and for charge carrier transport within the semiconductor and at the semiconductorelectrolyte interface. Interface states at the semiconductor-electrolyte interface were considered using an extended Schottky contact model. The numerical model was validated with current-voltage measurements using an n-type GaN photoanode immersed in $1 \mathrm{M} \mathrm{H}_{2} \mathrm{SO}_{4}$. Numerical design of experiments and parametric analysis were conducted using the validated model in order to identify and optimize the key factors for water-splitting photoelectrodes. The methodology, developed using an experimentally-validated numerical model coupled to statistical analysis, provides a general approach to identify and quantify the main material challenges and design considerations in working PEC devices. In the case of n-type GaN, the surface recombination, flatband potential, and doping concentration were identified as the most significant parameters for the photocurrent density.
\end{abstract}

\section{Introduction}

Photoelectrochemical (PEC) water-splitting devices require light absorbers, charge generators and separators, selective electrocatalysts, ion conducting electrolytes, and product separating semi-permeable membranes. Different approaches to integrated PEC water-splitting devices have been investigated..$^{1-3}$ Their key difference is the use of a solid-liquid junction (PEC cell), a solid-solid junction (PV cell), or combinations thereof, for charge separation. ${ }^{4,5}$ PV cell-based approaches allow for a separate optimization of the semiconductor and the electrolyte but suffer from more complex and expensive manufacturing and, usually, stability issues of the PV cell in the electrolyte. PEC cell-based approaches have the potential to be cheaper and provide tunable interface properties, but don't allow for separate treatment of the semiconductor, electrolyte, and sometimes the catalysts. The semiconductor-electrolyte interface is at the core of PEC water-splitting devices performance $^{6}$ and therefore requires special focus and investigation.

Research focusing on the energy and charge transfer phenomena at the illuminated semiconductor-liquid interface was pioneered by Marcus and Gerischer, and has considerably progressed over the decades. ${ }^{6-13}$ Electrochemistry at the semiconductor-liquid interface is often discussed in terms of the

Institute of Mechanical Engineering, École Polytechnique Fédérale de Lausanne, 1015 Lausanne, Switzerland. E-mail: sophia.haussener@epfl.ch; Tel: +4121693 3878

$\dagger$ Electronic supplementary information (ESI) available. See DOI: $10.1039 / \mathrm{c} 5 \mathrm{ta0} 07328 \mathrm{f}$
Marcus-Gerischer model ${ }^{10}$ which refers to ideal direct charge transfer. Nevertheless, deviations from the ideal charge transfer are observed in the presence of interface states as is observed for many semiconductor-electrolyte interfaces. Recently, numerical modelling of the charge transfer at the semiconductor-electrolyte interface and its coupling to multiphysical heat, mass, and charge transport phenomena in a complete PEC device has become of interest as it can provide insight into the coupled physical phenomena inaccessible to experiments. Models of charge transport in the semiconductor have enhanced the understanding of the energy band dynamics of photoabsorbers in direct contact with an electrolyte, ${ }^{\mathbf{1 4}}$ insight that can only be captured by numerical calculations. A 1dimensional model of a PEC water-splitting device integrating light absorption, charge transport in the semiconductor, charge transfer to the metallic catalyst, and charge transport in the electrolyte has quantified the dependency of the device performance on the choice of the light absorber. ${ }^{15}$ Theory and numerical modeling of charge transfer at semiconductor-catalyst interfaces for solar water-splitting have been developed to describe current-voltage behavior of semiconductor-catalystsolution systems with metallic, adaptive, and molecular catalysts. ${ }^{13}$ These models do not simultaneously study electromagnetic wave propagation and charge transport in the semiconductor and/or do not consider all types of relevant recombination phenomena, such as surface recombination, which can be a major loss at semiconductor-electrolyte interfaces. ${ }^{13,16,17}$ A detailed quantification and decoupling of the influence of the photon absorption, charge generation, charge 
transport and recombination, and interface transport and recombination phenomena is missing.

In this work, we uniquely combine numerical modeling, experimental measurements, and statistical analysis for the development of an accurate water-splitting photoelectrode performance model, identification and quantification of the key performance parameters, and subsequent proposition of material and device optimization. First, we introduce the numerical model, which combines electromagnetic wave propagation, charge generation and transport in the semiconductor, and charge transfer across the catalytically active semiconductor-electrolyte interface. The numerical model is then applied to a model system composed of a planar gallium nitride photoanode and platinum wire cathode immersed in 1 M sulfuric acid. The numerical model is completed with experimental investigations to determine missing material parameters of gallium nitride. The numerical model is subsequently experimentally validated using linear sweep voltammetry measurements. Statistical methods are used in combination with the validated model to identify the most important material and interface properties. Finally, parametric analyses are used to optimize identified key performance parameters of the water-splitting photoelectrode.

\section{Governing equations and methodology}

\subsection{Model domain and assumptions}

The modeled water-splitting photoelectrode consists of a planar photoanode (GaN) electrically connected to a wired counter electrode (Pt), both immersed in an electrolyte (sulfuric acid). The detailed arrangement of the planar photoelectrode, including substrate and highly-doped conduction layers, is depicted in Fig. 1a.

Electromagnetic wave (EMW) propagation is calculated in all components of the device (electrolyte and semiconductor), assuming solar irradiation at the top of the electrolyte and absorption at the back contact of the photoanode. The 2D model domain and its boundaries are indicated by a dotted frame in Fig. 1a. The radiation model attempts to be applicable to any type of photoelectrode or PEC device justifying the choice of the advanced light propagation model. The typically applied Beer-Lambert's law ${ }^{\mathbf{1 3 - 1 5}}$ limits the calculation of the charge generation rate to planar, homogeneous photoelectrodes, while detailed EMW propagation calculations provide solutions to the investigation and optimization of morphologically-complex, nano-structured, heterogeneous, or multi-component watersplitting photoelectrodes. Similarly, ray-tracing methods are also limited to cases where geometrical optics are valid, i.e. the thickness of the absorber is larger than the light wavelength. ${ }^{\mathbf{1 8}}$

Charge transport and conservation is solved only in the semiconductor component, utilizing dedicated boundary conditions to ensure the physical coupling to the counter electrode and the electrolyte; the front semiconductor domain boundary consists of a semiconductor-electrolyte interface, and the back semiconductor domain interface consists of a semiconductor-metal ohmic contact. The 1D model domain and its boundaries are indicated by a red line in Fig. 1. The governing equations of chemical species transport and reactions in the electrolyte are well known ${ }^{\mathbf{1 9 - 2 1}}$ and have been previously studied. ${ }^{22}$ Detailed analysis of the charge and species transport in the electrolyte was not considered in our study, assuming a highly conducting electrolyte with an excess availability of ions, no significant species concentration variations, and no mass transport limitations. Dissolved and gas-phase products

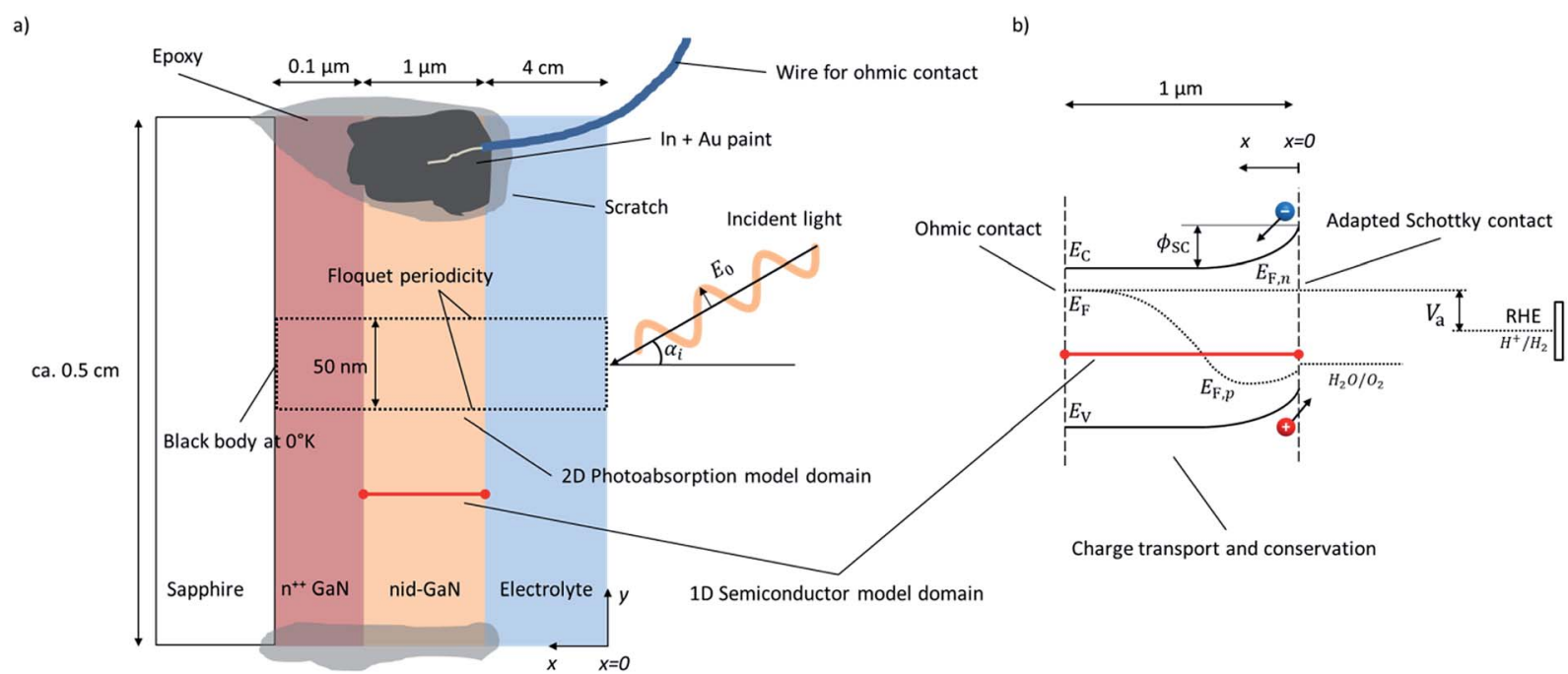

Fig. 1 (a) Scheme of the model domain (not to scale) of the photoanode (GaN) immersed in electrolyte including the 2D EMW propagation model domain (dotted) and boundary conditions, and the 1D semiconductor model domain (red line). (b) Detailed 1D semiconductor model domain and boundary conditions. $E_{\mathrm{C}}$ and $E_{\mathrm{V}}$ are the conduction and valence band potential levels, respectively. $\phi_{\mathrm{sc}}$ is the space charge layer potential, $E_{\mathrm{F}}, E_{\mathrm{F}, n}$ and $E_{\mathrm{F}, p}$ are the Fermi level, the electron and hole quasi-Fermi level potentials, respectively. $V_{\mathrm{a}}$ is the applied potential versus the reversible hydrogen electrode. 
such as oxygen and hydrogen were assumed to be quickly evacuated from the semiconductor-electrolyte interface. Generally, the electrolyte was assumed to be well stirred and purged. Flatband potentials were assumed to be unaffected by species adsorption at the semiconductor surface.

Our numerical model consists of two parts as depicted in Fig. 1: (i) a 2D model of the EMW propagation in the electrolyte and semiconductor that allows determination of the generation rate of electron-hole pairs in the semiconductor, and (ii) a 1D model of the charge transport and conservation in the semiconductor that allows determination of charge carrier concentrations, band positions, recombination rates, (photo)current, and potentials. The EMW propagation in $2 \mathrm{D}$ was developed to provide a general model enabling future study on more complex device structures. The advanced multi-dimensional EMW did not lead to significant additional computational expenses, as the electron-hole-pair generation rate was calculated only one time assuming that the complex refractive index was independent of the other semiconductor material properties. The $1 \mathrm{D}$ semiconductor model allowed for a computationally effective calculation and exploration of the material parameters of the semiconductor. Physical effects on charge transport due to multidimensionality of the sample were neglected and irrelevant for the planar sample with the highly conducting current collector.

The EMW propagation model assumed materials with a relative magnetic permeability of 1 and an electrical conductivity of zero. The various components were assumed rigid, homogeneous, and isotropic. Only steady state operation was considered, and time dependent effects such as photocorrosion were not considered.

2.1.1. Governing equations. The water-splitting photoelectrode model included EMW propagation, static and dynamic behavior of charge carriers in the semiconductor, and current transfer across the catalytically-active semiconductorelectrolyte interface.

Photoabsorption. The location-dependent charge carrier generation rate is calculated by solving the Maxwell's curl equations for each spectral band considered and the complex refractive index, $\tilde{n}(\omega)=n(\omega)-i k(\omega)$, as relevant material property, ${ }^{23}$

$$
\nabla \times(\nabla \times \boldsymbol{E}(\boldsymbol{x}, \omega))-k_{0}^{2} \tilde{n}(\omega)^{2} \boldsymbol{E}(\boldsymbol{x}, \omega)=0 .
$$

The optical power absorbed per unit volume is calculated from the electric field and the imaginary part of the complex permittivity,

$$
P_{\mathrm{abs}}(\boldsymbol{x}, \omega)=-\frac{1}{2} \omega|\boldsymbol{E}(\boldsymbol{x}, \omega)|^{2} \Im\{\varepsilon(\boldsymbol{x}, \omega)\},
$$

rather than by calculating the divergence of the Poynting vector, which is numerically less robust. The complex permittivity, $\varepsilon=$ $\varepsilon_{\mathrm{r}} \varepsilon_{0}=(n+i k)^{2} \varepsilon_{0}$, can be calculated from the complex refractive index and vacuum permittivity, $\varepsilon_{0}$. The total electron-hole generation rate, $G$, is calculated by integrating the spectral generation rate over the considered spectrum,

$$
G_{n}=G_{p}=G(\boldsymbol{x})=\int_{\infty}^{\omega_{\max }} P_{\mathrm{abs}}(\boldsymbol{x}, \omega) / h / \omega \mathrm{d} \omega,
$$

with the upper integration boundary $\omega_{\max } \geq E_{\text {gap }} / h$.

Charge transport and conservation. The static behavior of the charge carriers in the semiconductor is calculated by solving Poisson's equation, ${ }^{24}$

$$
\nabla \cdot\left(\varepsilon_{0} \varepsilon_{\mathrm{r}} \nabla \phi\right)=-\rho=q\left(n-p+N_{\mathrm{A}}^{-}-N_{\mathrm{D}}{ }^{+}\right),
$$

with the electron and hole density, $n$ and $p$, and the ionized acceptor and donor concentrations, $N_{\mathrm{A}}{ }^{-}$and $\mathrm{N}_{\mathrm{D}}{ }^{+}$.

The carrier density is given by integrating the product of the Fermi-Dirac distribution and the density of states over all possible states. For a non-degenerated semiconductor, i.e. when the Fermi level is at least $3 k_{\mathrm{B}} T$ away from either band edge, the Fermi-Dirac distribution can be replaced by the MaxwellBoltzmann distribution leading to electron and hole densities given by: ${ }^{\mathbf{2 4}}$

$$
\begin{aligned}
& n=N_{\mathrm{C}} \mathrm{e}^{-\left(E_{\mathrm{C}}-E_{\mathrm{F}}\right) / k_{\mathrm{B}} / T}, \\
& p=N_{\mathrm{V}} \mathrm{e}^{-\left(E_{\mathrm{F}}-E_{\mathrm{V}}\right) / k_{\mathrm{B}} / T} .
\end{aligned}
$$

The dynamic behavior of the carriers is calculated by solving the drift-diffusion equations for electrons and holes inside the semiconductor, ${ }^{24}$

$$
\begin{aligned}
& \boldsymbol{i}_{n}=\mu_{n} n \nabla E_{\mathrm{C}}+\mu_{n} k_{\mathrm{B}} T \nabla n, \\
& \boldsymbol{i}_{p}=\mu_{p} p \nabla E_{\mathrm{V}}-\mu_{p} k_{\mathrm{B}} T \nabla p .
\end{aligned}
$$

Isothermal device temperature and thermal equilibrium between the carrier and the lattice were assumed. The conduction band and valence energy levels, $E_{\mathrm{C}}$ and $E_{\mathrm{V}}$, are given by $E_{\mathrm{C}}=E_{\text {vaccum }}-q \chi$ and $E_{\mathrm{V}}=E_{\text {vaccum }}-q \chi-E_{\mathrm{g}}$ where $E_{\text {vaccum }}$ is the vacuum energy level and $\chi$ is the electron affinity. The total current density, $\boldsymbol{i}_{\text {tot }}$, is the sum of the electron and hole current densities. The steady state charge conservation is given by,

$$
\frac{1}{q} \nabla \cdot i_{n / p}=U_{n / p}
$$

where $U_{n / p}$ is the net electron or hole recombination rate,

$$
U_{n / p} \equiv R_{n / p}^{\mathrm{SRH}}+R_{n / p}^{\mathrm{rad}}+R_{n / p}^{\mathrm{Au}}+R_{\mathrm{s}, n / p}-G_{n / p}
$$

composed of three types of recombination in the bulk, i.e. Shockley-Read-Hall, radiative and Auger recombination, and one surface recombination type. The Shockley-Read-Hall recombination is given by,

$$
R_{n}^{\mathrm{SRH}}=R_{p}^{\mathrm{SRH}}=\frac{n p-\gamma_{n} \gamma_{p} n_{\mathrm{i}}^{2}}{\tau_{p}\left(n+n_{1}\right)+\tau_{n}\left(p+p_{1}\right)},
$$

with the bulk electron and hole lifetimes, $\tau_{n / p}$, and electron and hole degeneracy factors (assumed equal to one for non-degenerated semiconductors), $\gamma_{n}$ and $\gamma_{p} . n_{\mathrm{i}}=\sqrt{N_{\mathrm{C}} N_{\mathrm{V}}} \exp \left(-E_{\mathrm{g}} / 2 / k_{\mathrm{B}} / T\right)$ is the intrinsic density, and $N_{\mathrm{C}}$ and $N_{\mathrm{V}}$ the conduction and valence band densities of states, respectively. The electron and hole trap state densities are calculated according to

$$
n_{1}=\gamma_{n} n_{\mathrm{i}} \mathrm{e}^{\left(E_{\mathrm{t}}-E_{\mathrm{i}}\right) / k_{\mathrm{B}} / T},
$$




$$
p_{1}=\gamma_{p} n_{\mathrm{i}} \mathrm{e}^{-\left(E_{\mathrm{t}}-E_{\mathrm{i}}\right) / k_{\mathrm{B}} / T},
$$

where $E_{\mathrm{t}}-E_{\mathrm{i}}$ is the difference between the trap energy level and the intrinsic Fermi level, $E_{\mathrm{i}}=\left(E_{\mathrm{C}}+E_{\mathrm{V}}\right) / 2+k_{\mathrm{B}} T / 2 \ln \left(N_{\mathrm{V}} / N_{\mathrm{C}}\right)$. The electron and hole lifetimes in eqn (11) were exchanged by effective electron and hole lifetimes,

$$
\frac{1}{\tau_{\mathrm{eff}, n / p}}=\frac{1}{\tau_{n / p}}+\frac{1}{\tau_{\mathrm{s}, n / p}},
$$

which combines SRH bulk recombination and single level surface recombination. ${ }^{25,26}$ Consequently, eqn (11) was updated with an effective $\mathrm{SRH}$ recombination rate, $R_{\mathrm{eff}, n / p}^{\mathrm{SRH}}$, accounting also for surface recombination, and the surface recombination rate, $R_{\mathrm{s}, n / p}$, was removed. This simplifying approach was chosen since surface recombination can be expressed through a single trap level that follows the typical SRH recombination expression. ${ }^{27}$ The electron and hole surface lifetimes, $\tau_{\mathrm{s}, n / p}$, are material dependent properties.

The direct band-to-band radiative recombination,

$$
R_{n}^{\mathrm{rad}}=R_{p}^{\mathrm{rad}}=C_{\mathrm{dir}}\left(n p-\gamma_{n} \gamma_{p} n_{\mathrm{i}}^{2}\right)
$$

requires the direct recombination factor, $C_{\mathrm{dir}}$, as material property. Auger recombination involves three carriers and becomes important at high non-equilibrium carrier densities,

$$
R_{n}^{\mathrm{Au}}=R_{p}^{\mathrm{Au}}=\left(C_{\mathrm{aug}, n} n+C_{\mathrm{aug}, p} p\right)\left(n p-\gamma_{n} \gamma_{p} n_{\mathrm{i}}^{2}\right),
$$

where $C_{\mathrm{aug}, n}$ and $C_{\mathrm{aug}, p}$ are the Auger recombination factor material constants for electrons and holes.

2.1.2. Boundary conditions. The boundary conditions for the electromagnetic waves are front illumination with collimated and uniform irradiation at $x=0$, a black body at a temperature of $0 \mathrm{~K}$ at the back contact, and Floquet periodicity at the lateral walls (see Fig. 1). The spectral distribution of the illumination is detailed in Section 3.3.

The boundary conditions for the charge transport were an ohmic contact for the semiconductor-metal interface and an adapted Schottky contact model for the semiconductor-electrolyte interface, both detailed below. The boundary conditions presented here are at steady-state.

Ideal ohmic contact. The ideal ohmic contact (requiring local thermodynamic equilibrium at the contact) assumes that electron and hole quasi-Fermi levels are equal. Under this condition, eqn (4) describes charge neutrality and is used with $n_{0} p_{0}=$ $\gamma_{n} \gamma_{p} n_{\mathrm{i} \text {,eff }}^{2}$ to calculate the electron and hole equilibrium densities given as:

$$
\begin{gathered}
n_{\mathrm{eq}}=\frac{1}{2}\left(N_{\mathrm{D}}^{+}-N_{\mathrm{A}}^{-}\right)+\frac{1}{2} \sqrt{\left(N_{\mathrm{D}}^{+}-N_{\mathrm{A}}^{-}\right)^{2}+4 \gamma_{n} \gamma_{p} n_{\mathrm{i}}^{2}}, \\
p_{\text {eq }}=-\frac{1}{2}\left(N_{\mathrm{D}}^{+}-N_{\mathrm{A}}^{-}\right)+\frac{1}{2} \sqrt{\left(N_{\mathrm{D}}^{+}-N_{\mathrm{A}}^{-}\right)^{2}+4 \gamma_{n} \gamma_{p} n_{\mathrm{i}}^{2}} .
\end{gathered}
$$

The current, being conserved under steady-state, is determined by the current at the semiconductor-electrolyte interface, which must be equal to the current at the ohmic contact.

The electrostatic potential boundary condition for the ideal ohmic contact is given by:

$$
\phi=V_{\mathrm{a}} v s . \mathrm{RHE} .
$$

Under equilibrium and no applied potential, the potential for the ideal ohmic contact was chosen as zero vs. Reversible Hydrogen Electrode (RHE).

Adapted Schottky contact. An adapted Schottky contact was used for the determination of the current density at the semiconductor-electrolyte interface. Our adapted Schottky contact model accounts for interface states which influence the potential barrier height, $\phi_{\mathrm{B}}$, under dark and illumination, see Fig. 2. In the case of a majority carrier current, it also accounts for the interfacial potential drop distribution between the semiconductor space charge region (SCR) and the Helmholtz layer. Thus, the model enables prediction of band edge pinning or unpinning for majority carrier current.

An ideal Schottky describes the alignment of the Fermi level of the semiconductor with the dominant redox couple under
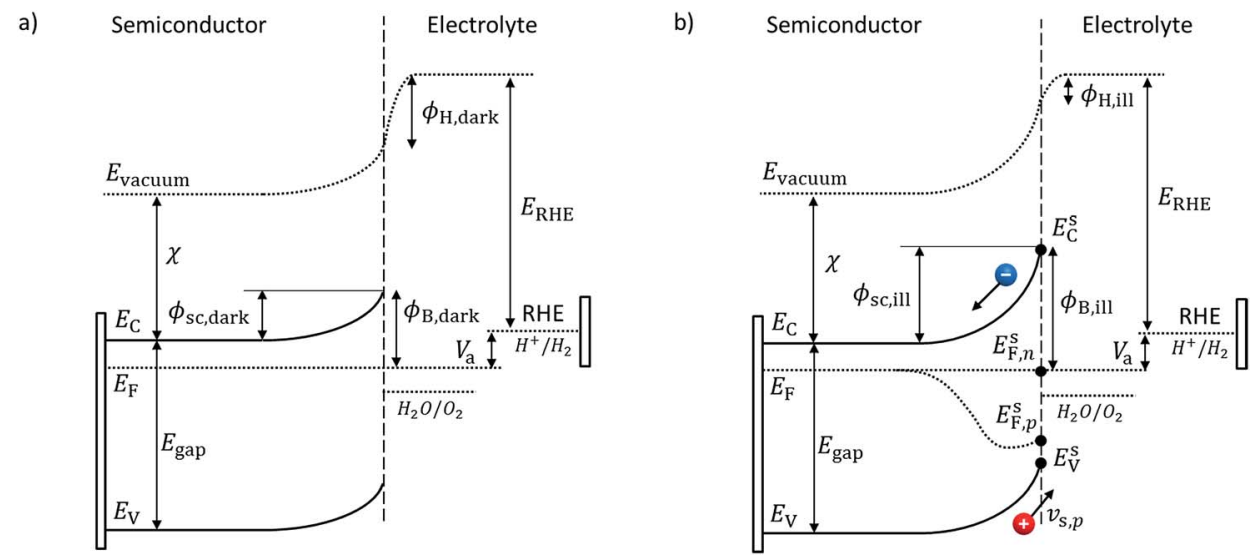

Fig. 2 Illustration of a n-type semiconductor-electrolyte interface under dark (a) and illumination (b). The applied potential $V_{a}$ is between the ohmic contact at the back of the photoelectrode and the reference electrode vs. RHE. The subscript "dark" stands for dark condition and "ill" for illuminated condition. The applied potential in this illustration is the same in the dark and illuminated conditions although it does not result in the same SCR. This situation is possible due to different flatband potentials in the dark and under illumination. 
equilibrium. This alignment provokes a depletion of negative charge (for n-type semiconductor), the SCR, which induces band bending. ${ }^{28}$ If large concentrations of interface states exist within the bandgap at the surface of the semiconductor-electrolyte interface, ${ }^{29}$ the Fermi level of the semiconductor might align with the interface states energy level instead and be "pinned" at the interface states' energy level. Upon illumination, the produced minority carriers might not cross the interface and therefore accumulate at the surface or get trapped by interface states ${ }^{30}$ which results in a change of the SCR potential, and therefore, the Helmholtz layer (HL) (see Fig. 2). This effect is called "unpinning of the band" and can be interpreted as the movement of the band edges. These complex effects were considered in our adapted Schottky contact by adapting the barrier height, $\phi_{\mathrm{B}}$, of the semiconductor SCR according to the flatband potential, $V_{\mathrm{FB}}$, under illumination or dark condition. The flatband potential refers to the situation where the applied potential is such that there is no band banding or charge depletion. ${ }^{31}$ The Mott-Schottky equation was used to determine the flatband potential of the semiconductor and therefore the barrier height. GaN has been reported to have strong interface states and therefore proves to be an excellent model material for our adapted Schottky contact model. ${ }^{32,33}$

The current density at the semiconductor-electrolyte interface was implemented as a Schottky contact mechanism with:

$$
\begin{gathered}
\boldsymbol{i}_{n} \cdot \hat{\mathbf{n}}=-q v_{\mathrm{s}, n}\left(n-n_{\mathrm{eq}}\right), \\
\boldsymbol{i}_{p} \cdot \hat{\mathbf{n}}=q v_{\mathrm{s}, p}\left(p-p_{\mathrm{eq}}\right), \\
i_{\mathrm{sc}}=\left(\boldsymbol{i}_{n}+\boldsymbol{i}_{p}\right) \cdot \hat{\mathbf{n}}=i_{\mathrm{H}},
\end{gathered}
$$

where $v_{\mathrm{s}, n}$ and $v_{\mathrm{s}, p}$ are the electrons and holes surface transfer kinetic velocities (called surface recombination velocities for a semiconductor-metal contact). $i_{\mathrm{sc}}$ is the total current density. Under current conservation, the current in the semiconductor must be equal to the current in the electrolyte, $i_{\mathrm{H}} \cdot n_{\mathrm{eq}}$ and $p_{\mathrm{eq}}$ are the carrier concentrations under equilibrium at the semiconductor-electrolyte interface and given by:

$$
\begin{gathered}
n_{\text {eq }}=N_{\mathrm{C}} \mathrm{e}^{-\phi_{\mathrm{B}} / V_{\mathrm{th}},} \\
p_{\text {eq }}=N_{\mathrm{V}} \mathrm{e}^{-\left(E_{\mathrm{gap}}-\phi_{\mathrm{B}}\right) / V_{\mathrm{th}}},
\end{gathered}
$$

where the barrier height is $\phi_{\mathrm{B}}=\phi_{\mathrm{sc}}+E_{\mathrm{C}}-E_{\mathrm{F}}$, as depicted in Fig. 2, and $V_{\text {th }}=k_{\mathrm{B}} T / q$.

The electron and hole densities at the semiconductor-electrolyte interface (as required in eqn (20) and (21)) are expressed in terms of the quasi-Fermi levels, using eqn (5) and (6):

$$
\begin{aligned}
& n=n_{\mathrm{eq}}+\Delta n=N_{\mathrm{C}} \mathrm{e}^{-\left(E_{\mathrm{C}}^{\mathrm{s}}-E_{\mathrm{F}, n}^{\mathrm{s}}\right) / k_{\mathrm{B}} T}, \\
& p=p_{\mathrm{eq}}+\Delta p=N_{\mathrm{V}} \mathrm{e}^{-\left(E_{\mathrm{F}, p}^{\mathrm{s}}-E_{\mathrm{V}}^{\mathrm{s}}\right) / k_{\mathrm{B}} T} .
\end{aligned}
$$

$\Delta n$ and $\Delta p$ are the additional carriers created by illumination, and $E_{\mathrm{F}, n}$ and $E_{\mathrm{F}, p}$ the quasi-Fermi levels of electrons and holes as depicted in Fig. 2. The superscript "s" stands for properties at the semiconductor-electrolyte interface. The electron and hole densities inside the semiconductor upon illumination are also given by eqn (25) and (26) with the corresponding quasi-Fermi levels and conduction and valence energy levels at a specific location.

Under dark condition and illumination, the SCR potential has to be known to determine the carrier densities which determine the current density (eqn (20) and (21)). In the case where the applied potential equals the flatband potential, $V_{\mathrm{a}}=$ $V_{\mathrm{FB}} v s$. RHE, there is no band banding and the SCR potential, $\phi_{\text {sc }}$, equals zero. By assuming that the applied potential drops only into the SCR potential, in dark condition with no applied potential ( $V_{\mathrm{a}}=0$ vs. RHE), the equilibrium SCR, $\phi_{\mathrm{sc} \text {,dark }}^{0}$, is equal to the flatband potential under dark:

$$
\phi_{\mathrm{sc}, \mathrm{dark}}^{0}=-V_{\mathrm{FB}, \text { dark }} v s . \mathrm{RHE} .
$$

The same assumption is used under illumination without an applied potential and therefore the SCR potential, $\phi_{\mathrm{sc} \text {,ill }}^{0}$, equals the flatband potential under illumination:

$$
\phi_{\mathrm{sc}, \text { ill }}^{0}=-V_{\mathrm{FB}, \text { ill }} v s . \mathrm{RHE} .
$$

In the case of an applied potential, the applied potential drops in the SCR and/or in the HL.

$$
V_{\mathrm{a}}=\Delta \phi_{\mathrm{sc}}+\Delta \phi_{\mathrm{H}}
$$

where $\Delta \phi_{\mathrm{sc}}$ and $\Delta \phi_{\mathrm{H}}$ are the SCR and HL potential difference between no applied potential and applied potential: $\Delta \phi_{\mathrm{sc}}=\phi_{\mathrm{sc}}$ $-\phi_{\mathrm{sc}}^{0}$ and $\Delta \phi_{\mathrm{H}}=\phi_{\mathrm{H}}-\phi_{\mathrm{H}}^{0}$.

We distinguished two different cases for the SCR potential under an applied potential: minority current and majority current.

Minority current. For a minority current, i.e. a hole current in the case of a n-type material, the HL potential difference, $\Delta \phi_{\mathrm{H}}$, is assumed to be negligible. ${ }^{28}$ The SCR potential under dark or illumination is given by:

$$
\begin{gathered}
\phi_{\mathrm{sc}, \mathrm{dark}}=\Delta \phi_{\mathrm{sc}, \mathrm{dark}}-\phi_{\mathrm{sc}, \mathrm{dark}}^{0}=V_{\mathrm{a}}-V_{\mathrm{FB}, \mathrm{dark}}, \\
\phi_{\mathrm{sc}, \mathrm{ill}}=\Delta \phi_{\mathrm{sc}, \mathrm{ill}}-\phi_{\mathrm{sc}, \mathrm{ill}}^{0}=V_{\mathrm{a}}-V_{\mathrm{FB}, \mathrm{ill}} .
\end{gathered}
$$

Generally, the minority current is influenced by the $\mathrm{HL}$ potential, species' concentration very close to the interface, and mass transport limits. Under such conditions, a detailed analysis based on the governing equation of chemical species transport and reactions in the electrolyte must be undertaken. ${ }^{19-21}$

Majority current. For a majority current, i.e. an electron current in the case of a n-type material, the applied potential is assumed to drop in the SC and $\mathrm{HL}$, and, consequently, $\Delta \phi_{\mathrm{H}}$ is assumed not equal to zero. We developed a simple analytical solution detailed in the ESI $\uparrow$ to determine $\Delta \phi_{\mathrm{H}}$ depending on the applied potential. In the case of a downward band bending for an n-type semiconductor, the HL potential is given by:

$$
\Delta \phi_{\mathrm{H}}\left(V_{\mathrm{a}}\right)=\frac{V_{\mathrm{th}} \ln \left(\frac{i_{\mathrm{H}_{2}}^{0}}{i_{n}^{0}}\right)+V_{\mathrm{a}}}{1+\alpha}<0 .
$$


where $i_{\mathrm{H}_{2}}^{0}$ is the exchange current density for the hydrogen evolution reaction, $i_{n}^{0}$ is the electron dark current densities given by $i_{n}^{0}=q v_{\mathrm{s}, n} n_{\mathrm{eq}}$, and $\alpha$ is the charge transfer coefficient typically around $0.5 .^{29}$

The SCR potential under dark condition and illumination in a case of a majority current in an n-type material is given by:

$$
\begin{gathered}
\phi_{\mathrm{sc}, \text { dark }}=\Delta \phi_{\mathrm{sc}, \text { dark }}-\phi_{\mathrm{sc}, \text { dark }}^{0}=V_{\mathrm{a}}-\Delta \phi_{\mathrm{H}}\left(V_{\mathrm{a}}\right)-V_{\mathrm{FB}, \mathrm{dark}} \\
\phi_{\mathrm{sc}, \mathrm{ill}}=\Delta \phi_{\mathrm{sc}, \mathrm{ill}}-\phi_{\mathrm{sc}, \text { ill }}^{0}=V_{\mathrm{a}}-\Delta \phi_{\mathrm{H}}\left(V_{\mathrm{a}}\right)-V_{\mathrm{FB}, \text { ill }}
\end{gathered}
$$

where the HL potential difference, $\Delta \phi_{\mathrm{H}}$, depends on the applied potential given by eqn (32).

The same approach can be used for an upward band bending in a p-type material in the case of a majority carrier current.

A commercial finite-element solver, Comsol Multiphysics $\odot,{ }^{34}$ was used to solve the coupled equations with the corresponding boundary conditions.

\subsection{Numerical design of experiment}

The numerical model described in the previous section depends on various parameters such as recombination rates, flatband potential, doping concentration, and charge transfer kinetics at the semiconductor-electrolyte interface, all of which significantly influence the efficiency of water-splitting photoelectrodes. We aimed at understanding the individual and coupled effects of these parameters on the performance of water-splitting photoelectrodes. A complete parameter sweep including all possible parameter combinations outgrew the resources $\left(2^{14}\right.$ combinations only considering the limiting parameter values). Consequently, we used a fractional factorial design (FFD) to statistically access the significance of the various parameters and their combinations. We chose a resolution five FFD study, reducing the number of combinations to $2^{14-6}$ while allowing for an understanding of the main effects and first level interactions. ${ }^{35}$ The data of the FFD were statistically analyzed using analysis of variance (ANOVA) ${ }^{36}$ providing the ability to comment on the statistical significance of a parameter effect. We ensured the residuals were random, independent, normally-distributed, and homogeneous. We used Bonferroni limit and $t$-student limits to assess the significance. $^{35}$

The FFD was used to screen for the most influential parameters on the photocurrent. Specifically, we chose to investigate the influence of the various parameters on the photocurrent at (i) a potential of $0.3 \mathrm{~V} v s$. RHE, and (ii) a potential of $1.23 \mathrm{~V} v s$. RHE. These potentials were chosen in order to investigate two situations were different characteristics might be dominating the performance. For example, at a potential of $1.23 \mathrm{~V} v$ s. RHE, i.e. at the thermodynamic oxygen evolution reaction potential, the recombination rate is expected to be relatively small while at $0.3 \mathrm{~V} v s$. RHE it starts to be the dominating effect on the photocurrent. These two cases can also represent two characteristic PEC cell designs: (i) a single cell design with one photo-absorber and a metallic counter electrode (the case at $0.3 \mathrm{~V} v$ s. RHE), or (ii) a tandem cell design where an additional photo-electrode provides additional potential (the case at $1.23 \mathrm{~V}$ vs. RHE).

The FFD only revealed the most significant parameters and (linear) interactions on the photocurrent. In order to gain information about the optimum values, we subsequently conducted a parametric study on the most relevant parameters to fully understand the effect of these parameters, their interactions, and their non-linear functional dependences.

\section{Application to gallium nitride}

A single layer of $1 \mu \mathrm{m}$ of non-intentionally doped (nid) gallium nitride (GaN) with wurtzite crystal structure was used as a model system. The planar GaN sample was immersed in $1 \mathrm{M} \mathrm{H}_{2} \mathrm{SO}_{4}$ with front illumination, as depicted in Fig. 1. A 2D model $(x-y$ plane) was used for EMW propagation and the same optical properties were used for nid-GaN and $\mathrm{n}^{++} \mathrm{GaN}$. The electrolyte was considered in the EMW propagation. The 1D semiconductor model was only considering nid-GaN since the $\mathrm{n}^{++}$ GaN was used to provide a conducting layer for the ohmic contact with a sheet resistance of approximately $50 \Omega \square^{-1}$. The 1D model accounted for a semi-infinite layer neglecting current variations in the other directions.

\subsection{Computational details}

3.1.1. Electromagnetic wave propagation. The electromagnetic wave propagation model was applied to the infinite slab of a GaN photoanode immersed into $1 \mathrm{M} \mathrm{H}_{2} \mathrm{SO}_{4}$ (assumed as water). Fig. 1 depicts the boundary conditions that were used in the computational modeling. The light was considered as a transverse electric wave. The EMW wavelengths were varied from $346.2 \mathrm{~nm}$ to $361.3 \mathrm{~nm}$ with $\Delta \lambda=0.27 \mathrm{~nm}$. The incident angle $\alpha_{\mathrm{i}}$ was set to $0^{\circ}$. Bloch-Floquet theory was assumed for the periodicity on both side of the computational domain which is typically used for infinite stab models where no boundary effects appear and where the phase shift is determined by a wave vector and the distance between the source and the destination. $^{37,38}$ Convergence was obtained with a direct MUMPS solver fully coupled for the corresponding variables. A relative tolerance of $10^{-3}$ in the three components of the electric field was used as convergence criteria. Mesh convergence was obtained for quadratic mesh elements with size ratio of 4 and element numbers, $n_{\mathrm{el}}$, depending on the irradiation wavelength, $\lambda$, the maximum refractive index $n_{\max }$ and the layer thickness, $d$ : $n_{\mathrm{el}}=n_{\mathrm{max}} d / 10 / \lambda$. The number of mesh elements perpendicularly to the direction of light propagation was fixed to 20 .

3.1.2. Semiconductor physics. A $1 D$ model was chosen for the solution of the semiconductor model with a single semiconductor layer and a semiconductor-electrolyte interface on the front side and an ohmic contact on the back side, as depicted in Fig. 1. The standard thickness of GaN was $1 \mu \mathrm{m}$. The model was calculated at steady-state. A uniform isothermal device temperature of $25{ }^{\circ} \mathrm{C}$ was assumed. A non-degenerate semiconductor was assumed using the Maxwell-Boltzmann distribution to calculate the carrier density. Convergence was obtained with a direct MUMPS solver fully coupled for the 
corresponding variables. A relative tolerance in the hole and electron concentrations and the electric potential of $10^{-3}$ was used as a convergence criteria. A segregated approach to solving electron and potential in one group, and electron, hole, and potential in the second group, appeared to be a good and fast alternative when convergence could not be obtained with the fully coupled approach. Mesh convergence was obtained for a mesh element number, $n_{\text {mesh }}=d / 20 \mathrm{~nm}$, with symmetric and linear mesh distributions and an element ratio of 7 . The symmetric distribution ensured a highly resolved mesh at each interface in the model.

\subsection{Material properties}

The spectrally resolved refractive index, $n$, and the extinction coefficient (i.e. imaginary part of refractive index), $k$, for wurtzite GaN were taken from Adachi. ${ }^{39}$ The complex refractive index data of $1 \mathrm{M} \mathrm{H}_{2} \mathrm{SO}_{4}$ was assumed to be of water and was taken from literature. ${ }^{40}$ The electrolyte height was set to $1 \mu \mathrm{m}$ in order to ensure correct calculation of the reflection behavior (for very small absorption in the wavelength range considered) while minimizing computational efforts.

$\mathrm{N}$-type GaN wurtzite crystal structure has been studied in the last decades for applications like LEDs. ${ }^{41}$ Parameters such as density of states for valence and conduction band, bandgap, electron affinity, relative permittivity, and recombination factors have been reported and are summarized in Table 1.

The carrier concentration-dependent electron mobility for ntype GaN was approximated as: ${ }^{49}$

$$
\mu_{n}\left(\mathrm{~cm}^{2} \mathrm{~V}^{-1} \mathrm{~s}^{-1}\right)=-98.02 \ln \left(N_{\mathrm{D}}^{+}\left(\mathrm{cm}^{-3}\right)\right)+4429.2,
$$

the temperature-dependent hole mobility was assumed similar to p-type GaN: ${ }^{50}$

$$
\mu_{p}\left(\mathrm{~cm}^{2} \mathrm{~V}^{-1} \mathrm{~s}^{-1}\right)=0.039(T(K))^{2}-26.945 T(K)+4709.7 .
$$

The nid-GaN used as a model system was a naturally n-type semiconductor with a donor concentration of $4 \times 10^{16} \mathrm{~cm}^{-3}$ determined through electrochemical impedance spectroscopy (see Section 3.4). Hence, the acceptor concentration was assumed to be zero. The flatband potentials, the effective lifetimes, and the dark currents are discussed in Section 3.4.

\subsection{Experimental details}

3.3.1. GaN sample preparation. GaN was deposited on a sapphire (0001) substrate using a Metal-Organic Vapor Phase Epitaxy (MOVPE) reactor of the laboratory LASPE, EPFL. A layer of $100 \mathrm{~nm}$ Si-doped GaN with doping concentration of $3 \times 10^{18}$ $\mathrm{cm}^{-3}$ was deposited on the sapphire substrate followed by $1 \mu \mathrm{m}$ of nid-GaN. The ohmic contact was made with indium in contact with the highly doped layer of GaN. Copper wires were fixed to indium using conductive liquid silver paint. GaN edges and ohmic contacts were protected from the electrolyte and light by applying white opaque epoxy. A photo of the GaN electrode is shown in Fig. 3.

3.3.2. Light source characteristics. A $1 \mathrm{~W}$ UV light-emitting diode (LED) with a nominal wavelength at $368 \mathrm{~nm}$ and with
Table 1 Material parameters and numerical values used for the model

\begin{tabular}{|c|c|c|}
\hline \multirow[t]{17}{*}{$\begin{array}{l}\text { Parameters for } \\
\text { semiconductor }\end{array}$} & Electron mobility, $\mu_{n}$ & $\begin{array}{l}682 \mathrm{~cm}^{2} \mathrm{v}^{-1} \mathrm{~s}^{-1} \\
(\mathrm{eqn}(35))\end{array}$ \\
\hline & Hole mobility, $\mu_{p}$ & $\begin{array}{l}143 \mathrm{~cm}^{2} \mathrm{~V}^{-1} \mathrm{~s}^{-1} \\
\text { (eqn (36)) }\end{array}$ \\
\hline & $\begin{array}{l}\text { Density of states, } \\
\text { valence band, } N_{\mathrm{V}}\end{array}$ & $\begin{array}{l}T^{3 / 2} \times 8.9 \times 10^{15} \mathrm{~cm}^{-3} \\
\text { (ref. } 42 \text { ) }\end{array}$ \\
\hline & $\begin{array}{l}\text { Density of states, } \\
\text { conduction band, } N_{\mathrm{C}}\end{array}$ & $\begin{array}{l}T^{3 / 2} \times 4.3 \times 10^{14} \mathrm{~cm}^{-3} \\
\text { (ref. } 43 \text { ) }\end{array}$ \\
\hline & $\begin{array}{l}\text { Direct recombination } \\
\text { factors, } C_{\mathrm{dir}}\end{array}$ & $\begin{array}{l}1.1 \times 10^{-8} \mathrm{~cm}^{3} \mathrm{~s}^{-1} \\
\text { (ref. } 44)\end{array}$ \\
\hline & Electron Shockley-Read-Hall & $1 \times 10^{-9} \mathrm{~s}$ \\
\hline & lifetime, $\tau_{n}$ & (ref. 45) \\
\hline & Hole Shockley-Read-Hall & $1 \times 10^{-9} \mathrm{~s}$ \\
\hline & lifetime, $\tau_{p}$ & (ref. 45) \\
\hline & Auger recombination & $2 \times 10^{-31} \mathrm{~cm}^{6} \mathrm{~s}^{-1}$ \\
\hline & factor, $C_{\mathrm{au}, n}=C_{\mathrm{au}, p}$ & (ref. 45 and 46 ) \\
\hline & Relative permittivity, $\varepsilon_{\mathrm{r}}$ & 8.9 (ref. 47$)$ \\
\hline & Band gap, $E_{\text {gap }}$ & $3.39 \mathrm{eV}$ (ref. 48) \\
\hline & Electron affinity, $\chi$ & $4.1 \mathrm{eV}$ (ref. 47 ) \\
\hline & Electron degeneracy & 1 \\
\hline & factor, $\gamma_{n}$ & \\
\hline & $\begin{array}{l}\text { Hole degeneracy } \\
\text { factor, } \gamma_{n}\end{array}$ & 1 \\
\hline \multirow{11}{*}{$\begin{array}{l}\text { Determined } \\
\text { parameters }\end{array}$} & Flatband potential & $-0.49 \mathrm{~V} v s . \mathrm{RHE}$ \\
\hline & under dark, $V_{\mathrm{FB}, \text { dark }}$ & $($ eqn $(37))$ \\
\hline & Flatband potential & $-0.66 \mathrm{~V} v s . \mathrm{RHE}$ \\
\hline & under dark, $V_{\mathrm{FB}, \text { ill }}$ & $($ eqn $(37))$ \\
\hline & Donor concentration, $N_{\mathrm{D}}^{+}$ & $\begin{array}{l}4 \times 10^{16} \mathrm{~cm}^{-3} \\
(\operatorname{eqn}(37))\end{array}$ \\
\hline & Electron effective & $8 \times 10^{-13} \mathrm{~s}$ \\
\hline & lifetime, $\tau_{\text {eff }}$ & $(\operatorname{eqn}(14))$ \\
\hline & Hole effective lifetime, $\tau_{\text {eff }}$ & $\begin{array}{l}8 \times 10^{-13} \mathrm{~s} \\
(\operatorname{eqn}(14))\end{array}$ \\
\hline & Electron dark current, $i_{n}^{0}$ & $\begin{array}{l}1.1 \times 10^{-10} \mathrm{~mA} \mathrm{~cm}^{-2} \\
(\text { eqn } \mathrm{S} 1 \text { ) }\end{array}$ \\
\hline & Hole dark current, $i_{p}^{0}$ & $\begin{array}{l}1.4 \times 10^{-41} \mathrm{~mA} \mathrm{~cm}^{-2} \\
(\text { eqn } \mathrm{S} 2)\end{array}$ \\
\hline & Trap energy level, $\Delta E_{\mathrm{t}}$ & $0 \mathrm{eV}($ eqn (12) and (13) \\
\hline Operating & Temperature, $T$ & $298.15 \mathrm{~K}$ \\
\hline
\end{tabular}
system made of nid-GaN 
high temporal stability was used as a light source. Back and front illuminations were possible with our new PEC experimental cell with a GE 124 quartz glass (94\% transmittance in the range of $300-750 \mathrm{~nm}$ ) of $2.54 \mathrm{~cm} \times 2.54 \mathrm{~cm}$ area placed on either side of the working electrode chamber (presented in Fig. 4). This additionally allowed for observation of the gas bubble formation. The LED's spectrum was measured using a spectral-stepping of $0.27 \mathrm{~nm}$ using a UV-Vis spectrometer (HR4000CG-UV-NIR from Ocean Optics). The total irradiance was measured with a thermal power sensor (S302C from Thorlabs). Both measurements were corrected for the absorption of the glass.

The spectral irradiance is shown in Fig. S1. $\dagger$ The total measured irradiance was $9.9 \mathrm{~mW} \mathrm{~cm} \mathrm{~cm}^{-2}$ at a distance of $4 \mathrm{~cm}$ from the LED of which $2.45 \mathrm{~mW} \mathrm{~cm}^{-2}$ could actually be absorbed by GaN with a bandgap of $3.39 \mathrm{eV}$ (equivalent to a band gap wavelength of $365.6 \mathrm{~nm}$ ).

3.3.3. PEC experimental setup and measurements. A newly developed type of PEC experimental cell presented in Fig. 4 was used for the experimental measurements. This cell was 3D printed using an acrylic-based photo-polymer, VeroWhite. The design of the cell allowed placement of the reference electrode very close to the working electrode and prevented gas crossovers between the working electrode and the counter electrode's chamber utilizing a Nafion membrane. Electrochemical experiments were carried out in a three-electrode setup to refer the potential of our measurements to the reversible hydrogen electrode. The electrodes were connected to a potentiostat (Bio-Logic VSP-300 controlled by the EC-lab software) for linear sweep voltammetry and impedance spectroscopy measurements. The reference electrode was $\mathrm{Ag} / \mathrm{AgCl}$ (sat. $\mathrm{KCl}$ ) and the counter electrode was Pt. The aqueous electrolyte solution was $1 \mathrm{M} \mathrm{H}_{2} \mathrm{SO}_{4}$.

The current-voltage curves were obtained using linear sweep voltammetry with a varying voltage rate of $10 \mathrm{mV} \mathrm{s}^{-1}$ (typically in a voltage range of -1 to $1.5 \mathrm{~V} v s$. RHE). The voltage rate of 10
$\mathrm{mV} \mathrm{s}^{-1}$ gave a stable steady-state current without any hysteresis on the photocurrent. Impedance spectra were measured at varying potentials which were scanned from -0.6 to $1 \mathrm{~V} v s$. RHE covering a frequency range of $300 \mathrm{mHz}$ to $1 \mathrm{MHz}$. The first run of the measurements are used (which ensured stable current conditions, see Fig. S2 $\dagger$ ) since GaN dissolves in the electrolyte after a few minutes under small current densities, i.e. around $0.5 \mathrm{~mA} \mathrm{~cm}^{-2}$.

\subsection{Experimental parameter value estimation}

The flatband potential and doping concentration were experimentally estimated by electrochemical impedance spectroscopy (EIS) using Mott-Schottky theory: ${ }^{28,31}$

$$
\frac{1}{C_{\mathrm{sc}}{ }^{2}}=\left(\frac{d Q_{\mathrm{sc}}}{\mathrm{d} \phi_{\mathrm{sc}}}\right)^{-2}=\frac{2}{\varepsilon_{0} \varepsilon_{r} q N_{\mathrm{D}}{ }^{+} A^{2}}\left(V_{\mathrm{a}}-V_{\mathrm{FB}}-\frac{k_{\mathrm{B}} T}{q}\right)
$$

where $C_{\mathrm{sc}}$ is the capacitance of the SCR, $A$ is the interfacial area, and $Q_{\mathrm{sc}}$ is the total charge enclosed within the semiconductor surface. Only frequencies higher than $50 \mathrm{~Hz}$ were considered in order to eliminate the effect of slow carrier processes. ${ }^{51}$ The Mott-Schottky plot for nid-GaN at different frequencies under dark condition is depicted in Fig. 5a. The Mott-Schottky plot shows a linear relationship between $1 / C_{\mathrm{sc}}{ }^{2}$ and $V_{\mathrm{a}}$, which ensured the pinning of the band edge. ${ }^{52}$ The Mott-Schottky plot showed only very little frequency dispersion in the frequency range of $500 \mathrm{~Hz}$ to $10 \mathrm{kHz}$ and therefore we did not fit the impedance spectra to an equivalent circuit. Instead, a linear function was fitted to the average Mott-Schottky plot for all frequencies depicted in Fig. 5a and used to determine the flatband potential and the doping concentration. The flatband potential under dark is $-0.49 \mathrm{~V}$ vs. RHE in $1 \mathrm{M} \mathrm{H}_{2} \mathrm{SO}_{4}$ and the doping concentration $4 \times 10^{16} \mathrm{~cm}^{-3}$. The Mott-Schottky plot also confirms that non-intentionally doped GaN is naturally ntype. The flatband potential is similar to previous values found in the literature $(-0.49 \mathrm{~V},-0.5 \mathrm{~V}$ and $-0.52 \mathrm{~V}$ vs. RHE (ref. 50,

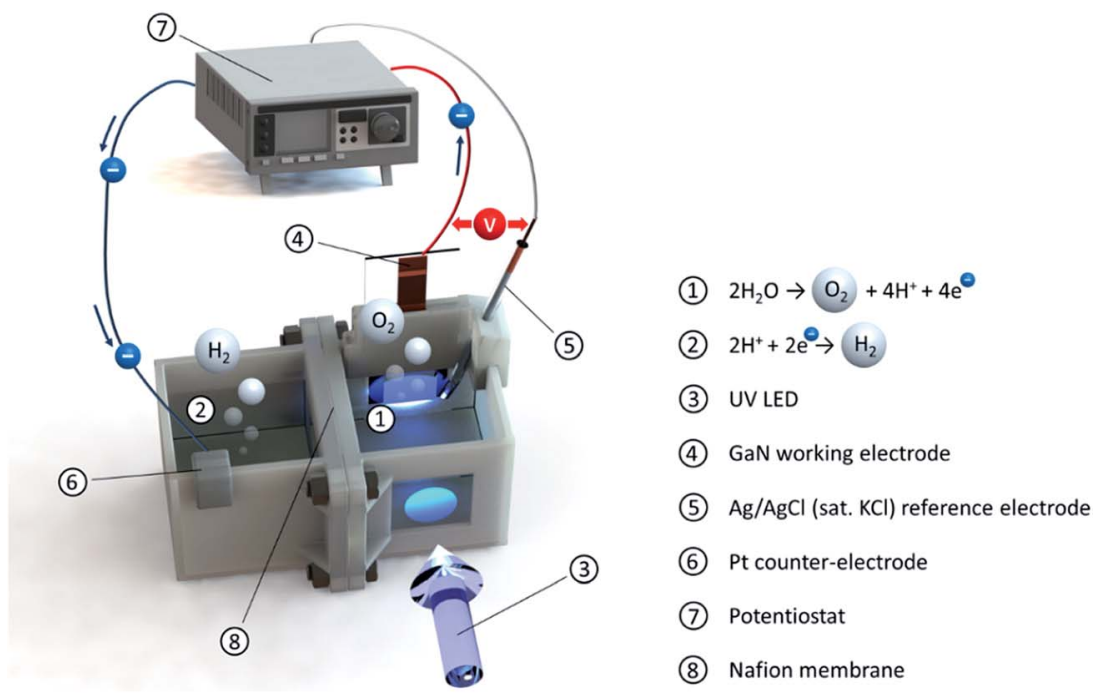

Fig. 4 Scheme of the experimental PEC water-splitting test cell connected to the potentiostat. The UV LED illuminates the working electrode through air, quartz glass, and electrolyte. 
a)

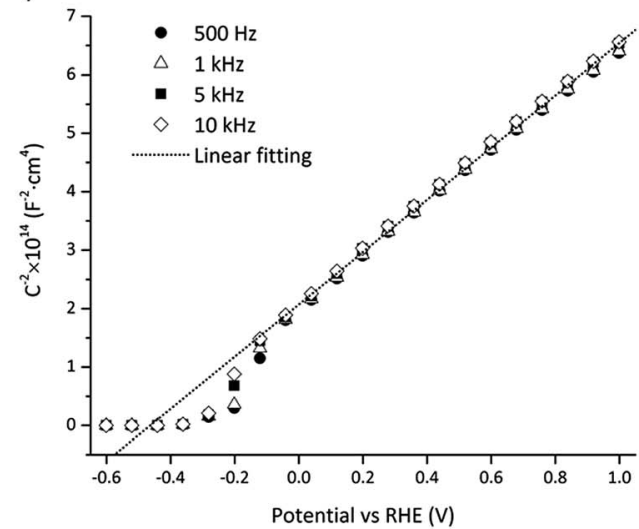

b)

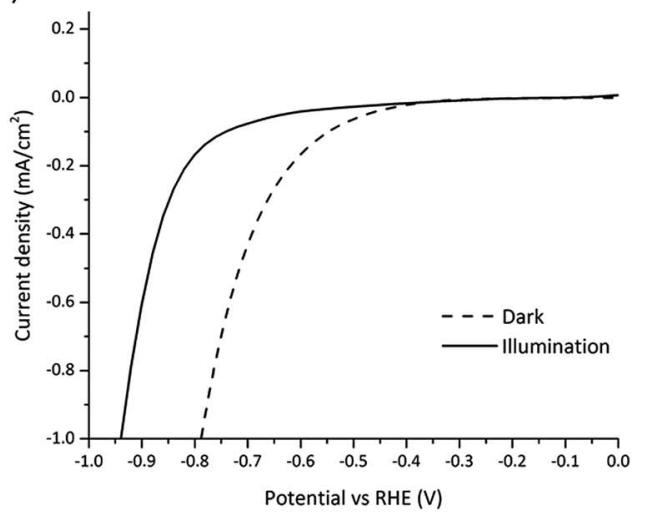

Fig. 5 (a) Mott-Schottky plot for four frequencies $(500 \mathrm{~Hz}, 1 \mathrm{kHz}, 5 \mathrm{kHz}, 10 \mathrm{kHz})$ of a $1 \mu \mathrm{m}$ thick nid-GaN sample immersed in 1 M H $\mathrm{H}_{2} \mathrm{SO}_{4}$ electrolyte under dark. (b) Experimental dark current density (dashed line) and photocurrent density (solid line) vs. RHE.

52 and 53)). The doping concentration of undoped GaN can vary significantly by unintentional incorporation of extrinsic impurities, mainly silicon and oxygen. Nevertheless, the doping concentration that we found is similar to nid-GaN with low impurities reported as $5 \times 10^{16} \mathrm{~cm}^{-3} \cdot{ }^{53}$

The small trough around $-0.5 \mathrm{~V}$ to $-0.1 \mathrm{~V} v s$. RHE depicted in Fig. $5 \mathrm{a}$ is assumed to result from interface states near the conduction band, i.e. the measured capacitance being the sum of the SC and the interface states capacitance. ${ }^{54}$ Interface states usually follow a Gaussian distribution which is in accordance with the observed trough (see Fig. 5a).

Interface states charging under illumination can cause a change in the flatband potential as previously mentioned and this effect has been observed for GaN in previous work. ${ }^{55}$ The shift in the flatband potential was measured by comparing the current for water reduction under dark and illumination depicted in Fig. 5b. The flatband potential shift was $-0.17 \mathrm{~V}$, which gave a flatband potential of $-0.66 \mathrm{~V} v s$. RHE under illumination.

\subsection{Numerical model validation}

Linear sweep voltammetry measurements and numerical simulations on GaN were used for the comparison between the steady-state numerical model and the experimental results. The surface charge transfer kinetic velocities for holes and electrons (see eqn (20) and (21)), electron and hole surface recombination lifetimes (see eqn (14)), and HER exchange current density (see eqn (32)) were estimated from linear sweep voltammetry by parameter fitting and are summarized in Table 2. The surface charge transfer velocities are smaller compared to typical semiconductor-metal interface velocities (usually in the range of $\left.1-10^{4} \mathrm{~m} \mathrm{~s}^{-1}\right)^{24}$ as the catalytically-driven electrochemical reaction slows down the charge transfer. The small surface recombination lifetimes indicated that surface recombination is a major loss in the case of nid-GaN. The exchange current density is three orders of magnitude below state-of-art HER catalysts. $^{22}$ The modeled case using parameter values indicated in Tables 1 and 2 is considered as our reference case.
Table 2 Material parameters determined by fitting to the linear sweep voltammetry measurement

\begin{tabular}{lll}
\hline Fitting parameters & $\begin{array}{l}\text { Electron surface } \\
\text { transfer kinetic velocity, } v_{\mathrm{s}, n}\end{array}$ & $1 \times 10^{-3} \mathrm{~m} \mathrm{~s}^{-1}$ \\
& $\begin{array}{l}\text { Hole surface } \\
\text { transfer kinetic velocity, } v_{\mathrm{s}, p}\end{array}$ & \\
& Electron surface & \\
& recombination lifetime, $\tau_{\mathrm{s}, n}$ & \\
& Hole surface \\
& recombination lifetime, $\tau_{\mathrm{s}, p}$ & \\
& & \\
& HER exchange \\
& current density, $i_{\mathrm{H}_{2}}^{0}$ & $1 \times 10^{-13} \mathrm{~s}$ \\
&
\end{tabular}

The only parameter that is changed in the experiment is the applied potential. Therefore, we compared the numerical current-potential dependency under dark and illumination with the experimental current-potential measurements. The measurements of $n-G a N$ were stable over eight minutes during linear sweep voltammetry and at photocurrent densities bellow one $\mathrm{mA} \mathrm{cm} \mathrm{cm}^{-2}$ (see Fig. S2 $\dagger$ ). We used the first linear sweep voltammetry measurements (from $1.5 \mathrm{~V}$ to $-1 \mathrm{~V} v s$. RHE, in about 2 minutes) for experimental-numerical comparison because GaN suffers from photocorrosion in acidic solutions although it is known to be resistant to corrosion. ${ }^{56,57}$ The numerically calculated dark current compares well to the experimentally measured current as depicted in Fig. 6. The calculated slope in the photocurrent is steeper at the onset potential than the measured one. This is explained by the losses within the highly doped GaN layer used for the charge collection, which were not accounted for in the model.

Two different dark current regimes can be distinguished in Fig. 6. Above the flatband potential $(-0.49 \mathrm{~V} v s$. RHE) the dark current is a minority current of only a few nA since nid-GaN is naturally n-type with a negligible amount of holes to actually enable the water oxidation. Indeed, the hole dark current density is only $1.4 \times 10^{-41} \mathrm{~mA} \mathrm{~cm}{ }^{-2}$ (see Table 1 ) producing a negligible dark current. Around and below the flatband potential, the current shifts to a majority current as there is no 


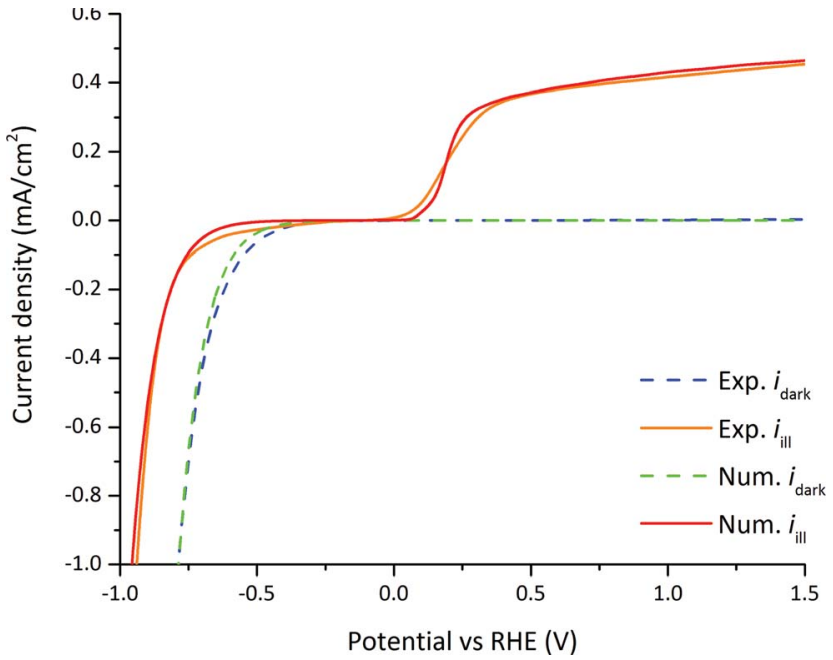

Fig. 6 Dark current densities (dashed lines) and photocurrent densities (solid lines) vs. RHE, and comparison between experimentally measured values and numerically calculated values.

more potential barrier with a downward bending band. In this case, the applied potential drops not only in the SC layer but also in the $\mathrm{H}$ layer in accordance with eqn (32).

Four different photocurrent regimes can be distinguished in Fig. 6. In the first regime at potentials between 0.4 to $1.5 \mathrm{~V} v s$. RHE, the photocurrent slightly decreases from the maximum photocurrent of $0.42 \mathrm{~mA} \mathrm{~cm}^{-2}$ at $1.5 \mathrm{~V} v s$. RHE to $0.37 \mathrm{~mA} \mathrm{~cm}^{-2}$ at $0.5 \mathrm{~V} v s$. RHE. In this regime the current density is a minority charge carrier current, holes are transferred from the semiconductor to the electrolyte for the water oxidation reaction. The applied potential and the band bending at the semiconductor-electrolyte interface provides an electric field large enough to allow for efficient charge separation. Recombination rates represent a $27 \%$ loss on the total photo-generation rate at $1.5 \mathrm{~V} v s$. RHE and $44 \%$ loss at $0.4 \mathrm{~V} v s$. RHE. This non-linear current drop is mainly associated to surface recombination since $\tau_{\mathrm{s}, n / p} \ll \tau_{\text {bulk,n/p}}$ and consequently $\tau_{\text {eff }, n / p} \approx \tau_{\mathrm{s}, n / p}$ (see eqn (14), and Tables 1 and 2) and is explained in detail in Section 3.7. Above 0 vs. RHE, the potential drops only in the semiconductor SCR.

In the second regime at potentials between 0.4 and $0 \mathrm{~V} v s$. RHE, the photocurrent abruptly decreases to zero at around $0 \mathrm{~V}$ $v s$. RHE. The electric field created by the potential and the band bending is not sufficient for charge separation and electronhole recombination starts to dominate. Recombination rates represent a $50 \%$ loss on the total photo-generation rate at $0.28 \mathrm{~V}$ vs. RHE and a $100 \%$ loss at $0.05 \mathrm{~V}$ vs. RHE.

In the third regime between potentials at $0 \mathrm{~V}$ and $-0.66 \mathrm{~V} v s$. RHE, the band bending starts to decrease until reaching the flatband potential conditions at $-0.66 \mathrm{~V} v s$. RHE. The recombination of electron and holes is complete and there is no photocurrent.

In the fourth regime between potentials -0.66 and $-1.0 \mathrm{~V} v s$. RHE, the current becomes a majority carrier current because of the downward band bending. Electrons are transferred from the semiconductor to the electrolyte for the water reduction reaction. The current decreases exponentially following eqn (21). The applied potential below the flatband potential drops not only in the SCR, but also in the HL layer in accordance with eqn (32). This behavior is consistent with the dark condition, only the flatband potential shifts between dark and illumination.

In addition to predicting the experimental values accessible by measurements, the benefit of the numerical model is that it allows for the prediction of the depth-dependent charge carrier generation, the depth-dependent electron and hole concentrations, and the depth-dependent energy levels of the quasi-Fermi levels, conduction band, and valence band in the semiconductor. Fig. S3-S5† show the distribution of these parameters for the reference case under illumination at $0 \mathrm{~V} v s$. RHE. The current is also depicted in Fig. 6 (red curve at $0 \mathrm{~V} v s$. RHE). The accessibility of such concentration and energy profiles greatly supports the understanding and interpretation of the observed current-voltage behavior.

\subsection{Numerical design of experiment}

The FFD was used to screen for the most influential semiconductor and semiconductor-electrolyte interface parameters on the photocurrent, i.e. fourteen parameters with two levels as presented in Table 3. The minimum and maximum values were carefully chosen to lie within realistic limits as otherwise no convergence in the numerical solution was achieved. The baseline parameters were required to lie between the upper and lower limits.

In order to ensure that the residuals were normally distributed, transformations in the photocurrent results were performed at both potentials investigated, i.e. $0.3 \mathrm{~V}$ and $1.23 \mathrm{vs}$. RHE. At $0.3 \mathrm{~V} v s$. RHE, a power function, $y^{\prime}=(y+k)^{\lambda}$, with $k=$

Table 3 Minimum and maximum parameter values used in the FFD under illumination

\begin{tabular}{|c|c|c|c|}
\hline Parameters & Unit & Minimum & Maximum \\
\hline $\begin{array}{l}\text { Electron surface transfer kinetic } \\
\text { velocity, } \nu_{\mathrm{s}, n}\end{array}$ & $\mathrm{~m} \mathrm{~s}^{-1}$ & $1 \times 10^{-4}$ & $1 \times 10^{-2}$ \\
\hline $\begin{array}{l}\text { Hole surface transfer kinetic } \\
\text { velocity, } \nu_{\mathrm{s}, p}\end{array}$ & $\mathrm{~m} \mathrm{~s}^{-1}$ & $1 \times 10^{-2}$ & $1 \times 10^{-1}$ \\
\hline Direct recombination factor, $C_{\mathrm{dir}}$ & $\mathrm{cm}^{3} \mathrm{~s}^{-1}$ & $1 \times 10^{-9}$ & $1 \times 10^{-7}$ \\
\hline Effective electron lifetime, $\tau_{\mathrm{eff}, n}$ & $\mathrm{~s}$ & $1 \times 10^{-13}$ & $1 \times 10^{-11}$ \\
\hline Effective hole lifetime, $\tau_{\text {eff }, p}$ & $\mathrm{~s}$ & $1 \times 10^{-13}$ & $1 \times 10^{-11}$ \\
\hline $\begin{array}{l}\text { Electron Auger recombination } \\
\text { factor, } C_{\text {aug }, n}\end{array}$ & $\mathrm{~cm}^{6} \mathrm{~s}^{-1}$ & $1 \times 10^{-33}$ & $1 \times 10^{-31}$ \\
\hline $\begin{array}{l}\text { Hole Auger recombination } \\
\text { factor, } C_{\text {aug }, p}\end{array}$ & $\mathrm{~cm}^{6} \mathrm{~s}^{-1}$ & $1 \times 10^{-33}$ & $1 \times 10^{-31}$ \\
\hline Donor concentration, $N_{\mathrm{D}}^{+}$ & $\mathrm{cm}^{-3}$ & $1 \times 10^{16}$ & $1 \times 10^{17}$ \\
\hline Flatband potential, $V_{\mathrm{FB}}$ & $\mathrm{V} v s . \mathrm{RHE}$ & -0.5 & -0.7 \\
\hline Relative permittivity, $\varepsilon_{\mathrm{r}}$ & - & 7 & 11 \\
\hline Semiconductor film thickness, $d$ & $\mu \mathrm{m}$ & 0.8 & 1.2 \\
\hline Hole mobility, $\mu_{p}$ & $\mathrm{~cm}^{2} \mathrm{~V}^{-1} \mathrm{~s}^{-1}$ & 50 & 200 \\
\hline $\begin{array}{l}\text { Effective density of states, valence } \\
\text { band, } N_{\mathrm{V}}\end{array}$ & $\mathrm{cm}^{-3}$ & $8 \times 10^{17}$ & $6 \times 10^{18}$ \\
\hline $\begin{array}{l}\text { Effective density of states, } \\
\text { conduction band, } N_{\mathrm{C}}\end{array}$ & $\mathrm{cm}^{-3}$ & $1 \times 10^{19}$ & $8 \times 10^{19}$ \\
\hline
\end{tabular}


0.0057 and $\lambda=0.61$ was used as the transformation function. At a potential of $1.23 \mathrm{~V} v s$. RHE, a power function with $k=0$ and $\lambda=1.99$ was used. At both potentials, the normal plot of the residuals indicated no abnormalities and the $R^{2}$ coefficients for a normal distribution were reasonable $(0.95$ for $1.23 \mathrm{~V} v s$. RHE and 0.88 for $0.3 \mathrm{~V} v s$. RHE). The residuals versus predicted values plots showed an approximately constant level of the studentized residuals across all predicted values and no outliers were found outside of the $95 \%$ confidence control limit in the studentized residuals versus run plot. Hence, the multiple regression model could be validated and the influence of each parameter could be safely investigated.

The Pareto charts depicted in Fig. 7 show the significant parameters or interactions, i.e. parameters or interactions with an effect above the Bonferroni limit. The most relevant factors at $1.23 \mathrm{~V} v s$. RHE are, from the largest to the smallest influence: $\tau_{\text {eff }, p}, \tau_{\text {eff, } n}$, the interaction between effective hole and doping concentration $\tau_{\text {eff, } n} \cdot N_{\mathrm{D}}{ }^{+}$, and ${N_{\mathrm{D}}}^{+}$(Fig. 7a). The other significant effects like the interaction between effective electron and hole lifetime, $\tau_{\mathrm{eff}, n} \cdot \tau_{\mathrm{eff}, p}$, and $\mu_{p}$ are also presented in Fig. 7a although their effects are lower compared to the effective lifetimes and the doping concentration. The bulk lifetimes are measured values ${ }^{45}$ which are intrinsic properties of GaN, but the lifetimes related to the surface recombination process depend on the semiconductor-electrolyte interface. The surface recombination lifetimes were more than three orders of magnitude lower than the bulk recombination and dominated the effective lifetimes (see Tables 1 and 2). Increasing the surface recombination lifetimes has a positive effect on the photocurrent (white bar in Fig. 7a) and can practically be obtained by surface passivation or by application of catalyst. The negative effect on photocurrent of the combined effective electron and hole lifetime $\left(\tau_{\mathrm{eff}, n} \cdot \tau_{\mathrm{eff}, p}\right)$ is explained by the non-linear dependence of these parameters on the photocurrent, inconsistent with the FFD assumption of linearity.

For the combined effect of effective electron lifetime and doping concentration $\left(\tau_{\mathrm{eff}, n} \cdot N_{\mathrm{D}}{ }^{+}\right)$, the negative effect of the doping concentration on the photocurrent is dominant. A more clear dependence of the most significant parameters on the photocurrent is investigated in the parametric study in the next section.

We used an effective lifetime combining surface recombination and SRH bulk recombination (see eqn (14)) and therefore the charge carrier concentration in the semiconductor is related to the surface lifetimes. Consequently, the observed strong dependence on doping concentration is a result of the effective lifetime assumption and not necessarily a physical result.

The most significant parameters influencing the photocurrent at $0.3 \mathrm{~V}$ vs. RHE are, from the largest to the smallest influence: $\tau_{\mathrm{eff}, p}, V_{\mathrm{FB}}$, and $\tau_{\mathrm{eff}, n}$ (Fig. 7b). The other significant effects are also indicated: $N_{\mathrm{D}}{ }^{+}$, the interaction between effective electron lifetime and doping concentration $\tau_{\text {eff, } n} \cdot{N_{\mathrm{D}}}^{+}$, and $v_{\mathrm{s}, p}$. According to FFD, it is beneficial for the photocurrent at $0.3 \mathrm{~V}$ $v s$. RHE to reduce the effective recombination of electrons and holes (dominated by surface lifetimes), and to increase the flatband potential.

\subsection{Parametric analysis on key factors}

A parametric study was done to precisely understand the functional dependence of the photocurrent on the most significant parameters according to FFD, i.e. the surface lifetimes of electrons and holes and the doping concentration at $1.23 \mathrm{~V} v$ s. RHE, and the surface lifetimes of the electrons and holes and the flatband potential at $0.3 \mathrm{~V}$ vs. RHE.

The influence of the hole surface lifetime on the photocurrent for varying applied potential is presented in Fig. 8. An increase of the hole surface lifetime has not only the beneficial effect of shifting the onset potential but also allows the further increase of the photocurrent at larger applied potentials. This unusual effect for an OER photocatalyst has been observed for hematite and $\mathrm{TiO}_{2}$ photoelectrodes whose surfaces were modified with phosphate ions. ${ }^{58,59}$ In both cases surface phosphate ions appeared to prolong the lifetime of holes on the surface. Interestingly, our numerical model is consistent with this effect using GaN as a reference model system for small hole surface lifetimes (around 1 ps). At larger hole surface lifetimes
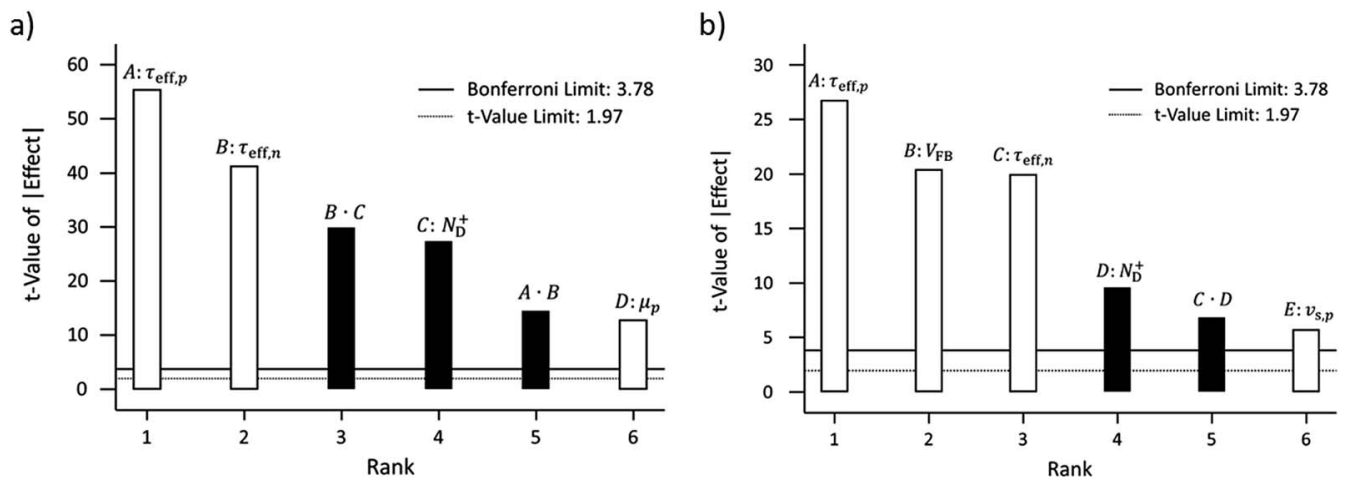

Fig. 7 Pareto plots indicating the significance of the photocurrent response at a potential of $1.23 \mathrm{~V}(\mathrm{a})$ and $0.3 \mathrm{~V}$ (b) vs. RHE calculated utilizing the FFD of experiment. White bars indicate an increase and black bars a decrease, respectively, of the photocurrent when increasing the corresponding parameter. $\tau_{\mathrm{eff}, n}$ and $\tau_{\mathrm{eff}, p}$ are effective electron and hole lifetimes, $N_{\mathrm{D}}{ }^{+}$is the doping concentration, and $\mu_{p}$ is the hole mobility, $V_{\mathrm{FB}}$, is the flatband potential, and $\nu_{\mathrm{s}, p}$ is the hole surface transfer kinetic velocity. 


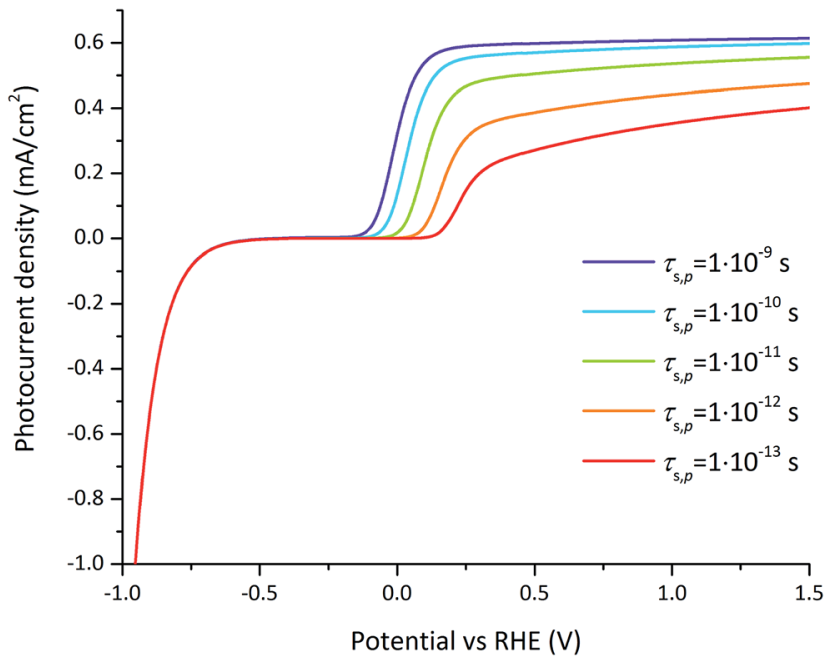

Fig. 8 Photocurrent-voltage curves for varying hole surface lifetimes for the reference case (parameters indicated in Tables 1 and 2). For large hole surface lifetimes (above 0.1 to $1 \mathrm{~ns}$ ), surface recombination is negligible at large applied potentials. For small hole surface lifetimes (around 1 ps), the photocurrent is still affected by surface recombination at large potentials.

(above 0.1 to $1 \mathrm{~ns}$ ), the current-potential dependency follows the expected behavior, namely that surface recombination is negligible for an applied potential above $0.2 \mathrm{~V} v s$. RHE.

The dependence of the photocurrent densities at $1.23 \mathrm{~V} v s$. RHE on surface lifetimes and doping concentration are depicted in Fig. 9. At low doping concentration, i.e. $1 \times 10^{16} \mathrm{~cm}^{-3}$ for $\mathrm{GaN}$, an increase of the effective hole and electron surface lifetimes results in an increase in photocurrent (Fig. 9). If the electron surface lifetime is large enough, e.g. $1 \mathrm{~ns}$, the hole surface lifetime has a less significant impact on the photocurrent; a relative increase by $9.5 \%\left(0.06 \mathrm{~mA} \mathrm{~cm}^{-2}\right)$ for 4 orders of

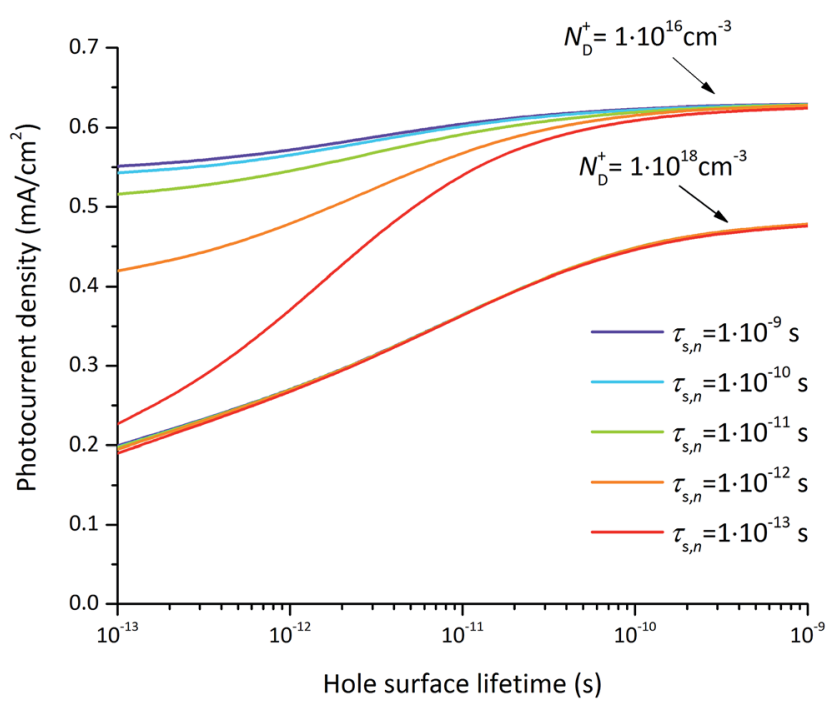

Fig. 9 Photocurrent density at $1.23 \mathrm{~V}$ vs. RHE as a function of the hole surface lifetime for various electron lifetimes $\left(\tau_{\mathrm{s}, n}=10^{-9}, 10^{-10}, 10^{-11}\right.$, $\left.10^{-12}, 10^{-13} \mathrm{~s}\right)$ and two doping concentrations $\left(N_{\mathrm{D}}^{+}=10^{16} \mathrm{~cm}^{-3}\right.$ and $N_{\mathrm{D}}^{+}=10^{18} \mathrm{~cm}^{-3}$ ). magnitude difference of hole surface lifetime is observed (violet line in Fig. 9). For low electron surface lifetime, e.g. $0.1 \mathrm{ps,}$ increasing the hole surface lifetime from $1 \mathrm{ps}$ to $0.1 \mathrm{~ns}$ increases the photocurrent by $0.34 \mathrm{~mA} \mathrm{~cm}^{-2}$, which represents a relative increase of $58 \%$. Increasing the doping concentration to $1 \times$ $10^{18} \mathrm{~cm}^{-3}$ results in a photocurrent which is not affected by the electron surface lifetime (Fig. 9). On the other hand, the hole surface lifetime is still significant at these larger doping concentrations, i.e. increasing the hole surface lifetime from 1 ps to $1 \mathrm{~ns}$ increases the photocurrent by $0.21 \mathrm{~mA} \mathrm{~cm}^{-2}$.

The photocurrent, being a minority current, is generally more influenced by the hole surface lifetime. Especially at large hole surface lifetimes, the influence of electron surface lifetime becomes negligible. The insensitivity of the photocurrent on electron surface lifetime at high doping concentrations results from the dominating terms in the recombination, namely the electron concentration and hole surface lifetime (see eqn (11)). As mentioned, the photocurrent results from the combined influence of the numerical value of the hole surface lifetime, electron surface lifetime, and doping concentration. Therefore under low doping and low hole surface lifetime the electron surface lifetime can still have an impact on the photocurrent at $1.23 \mathrm{~V}$ which appears counterintuitive.

The doping concentration-dependent current-voltage behavior under illumination is depicted in Fig. 10. An optimal doping concentration is found at a value of $N_{\mathrm{D}}^{+}=10^{16} \mathrm{~cm}^{-3}$ above about $0.2 \mathrm{~V} v$ s. RHE as depicted in Fig. S6. $\uparrow$ The optimum is caused by different and opposing effects. On one hand a decrease of the doping concentration leads to a lower Fermi level. Since the Fermi level aligns with the interface states located at the semiconductor-electrolyte interface, it leads to a higher band bending at the semiconductor-electrolyte interface and therefore a positive shift of the onset potential and an increase of the photocurrent (can be clearly observed on the negative potential side). On the other hand, the recombination rate increases with larger doping concentrations simply because of the increased electron concentration which reduces the photocurrent. At very low doping concentrations, the semiconductor is completely depleted and there is no band bending but instead a linearly increasing band potential throughout the entire semiconductor. By assuming locally a constant potential change and by integrating the drift-diffusion equations (eqn (7) and (8)) within the semiconductor, the current-potential relation is predicted by Ohm's law. This situation is depicted in Fig. 10 at a doping concentration of $N_{\mathrm{D}}{ }^{+}=10^{14} \mathrm{~cm}^{-3}$ where the photocurrent versus potential starts to show a linear trend. These effects must be considered when optimizing the photocurrent density by variations in doping concentration and operating potential. For example at an operating potential of 0.3 $\mathrm{V} v s$. RHE, the photocurrent density increases by $0.25 \mathrm{~mA} \mathrm{~cm}^{-2}$ by changing the doping concentration from $10^{14} \mathrm{~cm}^{-3}$ to $10^{16}$ $\mathrm{cm}^{-3}$ which represents a relative gain of $69 \%$.

The photocurrent densities at $0.3 \mathrm{~V}$ vs. RHE depend most significantly on the flatband potential, electron lifetime, and hole surface lifetime. The variation of the flatband potential gives rise to a shift of the onset potential (see Fig. $\mathrm{S} 7 \dagger$ ). A more complex behavior is observed when also varying the surface 


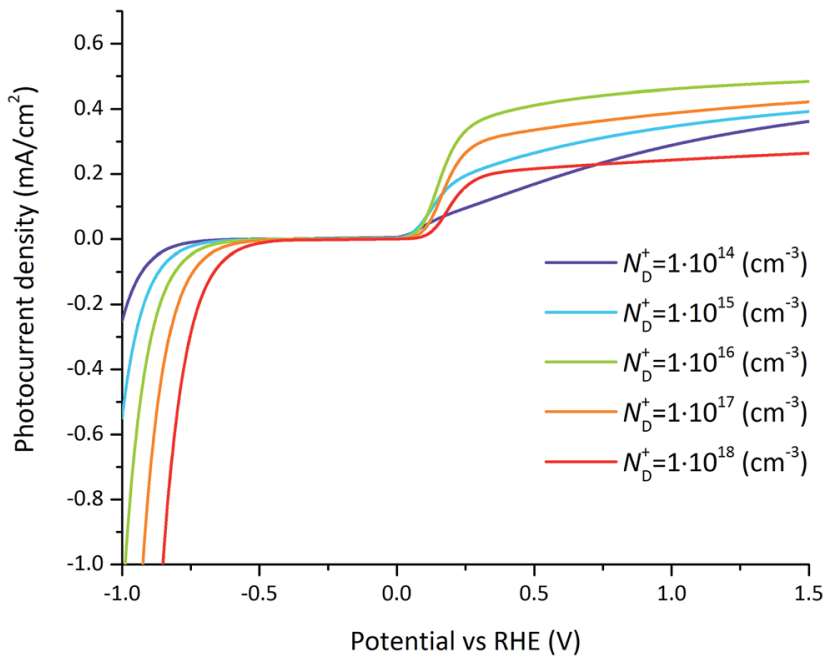

Fig. 10 Photocurrent-voltage curves for varying doping concentration for the reference case (parameters indicated in Tables 1 and 2). For small doping concentrations (bellow $10^{14} \mathrm{~cm}^{-3}$ ), the photocurrentpotential relation is linear. For intermediate doping concentrations (around $10^{16} \mathrm{~cm}^{-3}$ ), the photocurrent shows an optimum at which the band bending is maximized and recombination is reasonable. At large doping concentrations (above $10^{18} \mathrm{~cm}^{-3}$ ) recombination dominates.

lifetimes as depicted in Fig. 11. At a flatband potential of $-0.5 \mathrm{~V}$, the band bending is reduced resulting in a decreased electric field and consequently a decreased hole transfer from the semiconductor to the electrolyte (the photocurrent). Since the electric field is lower, recombination becomes the dominating loss which is directly related to the hole and electron surface lifetimes (Fig. 11a).

A significant effect of the hole surface lifetime on the photocurrent is observed at a flatband potential of $-0.5 \mathrm{~V} v$ s. RHE (Fig. 11a). At low electron lifetime, i.e. $0.1 \mathrm{ps}$, the photocurrent increases from $0 \mathrm{~mA} \mathrm{~cm}{ }^{-2}$ to $0.56 \mathrm{~mA} \mathrm{~cm}{ }^{-2}$ when changing the hole surface lifetime by four orders of magnitude, i.e. from $0.1 \mathrm{ps}$ to $1 \mathrm{~ns}$. Even at higher electron surface lifetime, i.e. $1 \mathrm{~ns}$, the photocurrent increases from $0.24 \mathrm{~mA} \mathrm{~cm}{ }^{-2}$ to $0.54 \mathrm{~mA} \mathrm{~cm}^{-2}$ (a relative increase of $55 \%$ ) when increasing the hole surface lifetime from $0.1 \mathrm{ps}$ to $1 \mathrm{~ns}$. The behavior is similar at a flatband potential of $-0.7 \mathrm{~V} v$ s. RHE (Fig. 11b), although the electron and hole surface lifetimes have a smaller impact: the photocurrent increases by $0.4 \mathrm{~mA} \mathrm{~cm}^{-2}$ (from $0.18 \mathrm{~mA} \mathrm{~cm}^{-2}$ to $0.58 \mathrm{~mA} \mathrm{~cm}^{-2}$, i.e. a relative increase of $69 \%$ ) when increasing the hole surface lifetime from 0.1 ps to $1 \mathrm{~ns}$ at an electron surface lifetime of 0.1 ps. At an electron surface lifetime of $1 \mathrm{~ns}$, the photocurrent increases by $0.29 \mathrm{~mA} \mathrm{~cm}^{-2}$ (a relative increase of $48 \%$ ) when increasing the hole surface lifetime from 0.1 ps to $1 \mathrm{~ns}$.

\section{Summary and conclusion}

A multi-physics model of a semiconductor water-splitting photoelectrode immersed in electrolyte was developed. The model coupled electromagnetic wave propagation, charge carrier generation and transport, and charge transfer at the semiconductor-electrolyte interface. The model provided, among others, spatially resolved energy band diagrams, charge carrier concentrations, and generation and recombination profiles in the semiconductor. The model incorporated an adapted Schottky contact at the semiconductor-electrolyte interface, accounting for pinning and unpinning of the band edges and for potential drop within the SCR as well as the HL. The interface model presented allows for a straightforward extension to semiconductor-catalyst-solution systems with metallic, adaptive and molecular catalysts by using the boundary conditions presented in the work of Mills et al. ${ }^{13}$ The HL played only a role in charge transfer at the semiconductor-electrolyte interface for majority carrier currents. In this case, the potential distribution between the HL and the SCR was determined by a newly derived analytical solution.

The numerical model was applied to our model system composed of a non-intentionally doped n-type gallium nitride (GaN) with wurtzite crystal structure photoelectrode layer immersed in $1 \mathrm{M}$ sulfuric acid. GaN was chosen here as it allows for unbiased photoelectrochemical water-splitting. Additionally, GaN has been shown to be considerably resistant to corrosion in many solutions in the dark ${ }^{49}$ although it gradually dissolves under illumination. GaN has been known to have
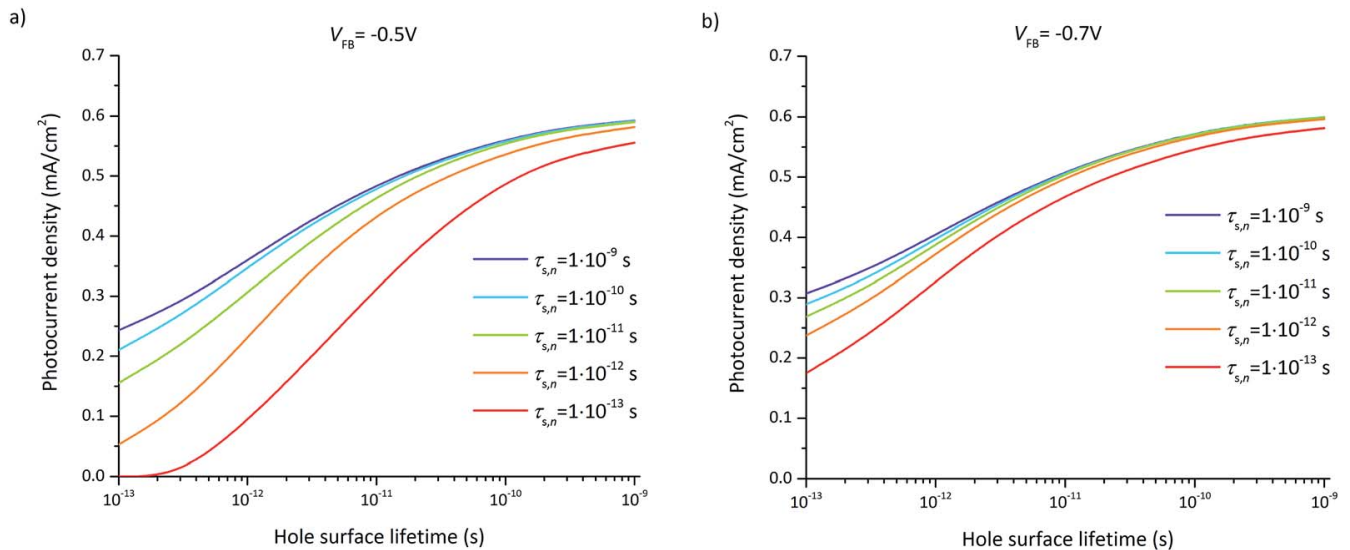

Fig. 11 Photocurrent density at $0.3 \mathrm{~V}$ vs. RHE as a function of the hole surface lifetime for various electron lifetimes $\left(\tau_{\mathrm{s}, n}=10^{-9}, 10^{-10}, 10^{-11}\right.$, $10^{-12}, 10^{-13} \mathrm{~s}$ ) and two flatband potentials: (a) $V_{\mathrm{FB}}=-0.5 \mathrm{~V} v \mathrm{~s}$. $\mathrm{RHE}$, and (b) $V_{\mathrm{FB}}=-0.7 \mathrm{~V} v \mathrm{~s}$. RHE. 
interface states, therefore flatband potentials under dark and illumination were experimentally determined by electrochemical impedance spectroscopy using Mott-Schottky theory. Flatband potentials under dark and illumination were found to be $-0.49 \mathrm{~V}$ and $-0.66 \mathrm{~V} v s$. RHE. Impedance spectroscopy was also used to estimate the intrinsic doping concentration of nidGaN, estimated as $4 \times 10^{16} \mathrm{~cm}^{-3}$. Linear sweep voltammetry was then used to determine photocurrent response as a function of the applied potential to which the modeled photocurrent-potential response was compared in order to validate the multi-physics model.

The multi-physics model allowed representation of numerous semiconductor materials with numerous semiconductor-electrolyte interface properties such as electron and hole mobilities, surface lifetimes, flatband potential, permittivity, doping concentration, bulk SRH recombination, and hole and electron interface kinetics. The large number of relevant material and interface characteristics renders the identification of the most significant parameter(s) challenging. Statistical tools provide a pathway for solving this challenge as demonstrated in this study. The validated model was used in a FFD of experiment to statistically identify the most significant material and interface parameters and device dimensions on the photocurrent. Key factors were identified at two different potentials: $0.3 \mathrm{~V} v s$. RHE and $1.23 \mathrm{~V} v$ s. RHE. Hole and electron surface lifetimes and doping concentration appeared to be the most significant factors at $1.23 \mathrm{~V} v s$. RHE. At $0.3 \mathrm{~V} v s$. RHE, hole and electron surface lifetimes and flatband potential were the most significant factors. The statistically identified most significant parameters were further investigated and theoretically optimized in a detailed parameter study. The parametric analysis provided quantifiable effects and functional dependence of the photocurrent on the predominant factors previously determined by FFD analysis.

The developed methodology uses an experimentally-validated numerical model and statistical analysis to provide understanding of the performance of water-splitting photoelectrodes. It allows for the identification of the most significant parameters on performance. Subsequent in-depth parametric analysis of the most significant parameters allows for the quantification of their effect and subsequent optimization of the device for maximum performance. The presented methodology provides a general approach to identify and quantify main material challenges and design considerations in functioning water-splitting photoelectrodes. The predictive character of the validated model can be further exploited with confidence to approach and investigate morphologically complex electrodes and material classes for which research-related questions are not yet answered.

\section{Conflict of interest}

The authors declare no competing financial interest.

\section{Acknowledgements}

The authors would like to acknowledge the funding from the National Science Foundation under Grant \#200021_159547. The authors would like to thank Dr Thomas Moehl (LPI at EPFL), for helpful discussions on electrochemical impedance spectroscopy, Dr Jean-François Carlin (LASPE at EPFL) for the deposition of nid-GaN on sapphire substrate, and Dr Mikaël Dumortier (LRESE at EPFL) and Dr Simone Pokrant (EMPA) for fruitful discussions.

\section{References}

1 K. Sivula, Chimia, 2013, 67, 155-161.

2 M. A. Modestino and S. Haussener, Annu. Rev. Chem. Biomol. Eng., 2014, 6, 13-34.

3 T. J. Jacobsson, V. Fjällström, M. Edoff and T. Edvinsson, Energy Environ. Sci., 2014, 7, 2056-2070.

4 M. G. Walter, E. L. Warren, J. R. McKone, S. W. Boettcher, Q. Mi, E. a. Santori and N. S. Lewis, Chem. Rev., 2010, 110, 6446-6473.

5 A. C. Nielander, M. R. Shaner, K. M. Papadantonakis, S. a. Francis and N. S. Lewis, Energy Environ. Sci., 2015, 8, 16-25.

6 A. J. Nozik and R. Memming, J. Phys. Chem., 1996, 100, 13061-13078.

7 J.-N. Chazalviel, J. Electrochem. Soc., 1982, 129, 963-969.

8 D. Vanmaekelbergh, Electrochim. Acta, 1997, 42, 1121-1134.

9 D. Vanmaekelbergh, Electrochim. Acta, 1997, 42, 1135-1141.

10 M. N. Latto, G. Pastor-Moreno and D. J. Riley, Electroanalysis, 2004, 16, 434-441.

11 J. Reichman, Appl. Phys. Lett., 1980, 36, 574-577.

12 R. Memming, Semiconductor Electrochemistry, Wiley-VCH, Weinheim, 2001.

13 T. J. Mills, F. Lin and S. W. Boettcher, Phys. Rev. Lett., 2014, 112, 148304.

14 P. Cendula, S. D. Tilley, S. Gimenez, J. Bisquert, M. Schmid, M. Grätzel and J. O. Schumacher, J. Phys. Chem. C, 2014, 118, 29599-29607.

15 A. Berger and J. Newman, J. Electrochem. Soc., 2014, 161, 3328-3340.

16 F. E. Osterloh, Chem. Soc. Rev., 2013, 42, 2294-2320.

17 T. Lopes, L. Andrade, F. Le Formal, M. Gratzel, K. Sivula and A. Mendes, Phys. Chem. Chem. Phys., 2014, 16, 16515-16523.

$18 \mathrm{R}$. Siegel and J. Howell, Thermal radiation heat transfer, Taylor \& Francis, London, 4th edn, 2001.

19 J. Newman and K. E. Thomas-Alyea, Electrochemical Systems, John Wiley \& Sons, Inc., 3rd edn, 2001.

20 A. J. Bard and L. R. Faulkner, Electrochemical Methods: Fundamentals and Application, John Wiley \& Sons, Inc., 2nd edn, 2001.

$21 \mathrm{~K}$. J. Vetter, Electrochemical Kinetics Theoretical and Experimental Aspects, Academic Press Inc., 1967.

22 S. Haussener, C. Xiang, J. M. Spurgeon, S. Ardo, N. S. Lewis and A. Z. Weber, Energy Environ. Sci., 2012, 5, 9922-9935.

23 D. M. Pozar, Microwave Engineering, 4th edn, 2012.

24 K. K. N. S. M. Sze, Physics of semiconductor devices, Wiley-inte, 2007.

25 K. Gullbinas, V. Grivickas, H. P. Mahabadi, M. Usman and A. Hallen, Mater. Sci., 2011, 17, 119-124.

26 A. B. Sproul, J. Appl. Phys., 1994, 76, 2851-2854. 
27 D. J. Michalak, F. Gstrein and N. S. Lewis, J. Phys. Chem. C, 2008, 112, 5911-5921.

28 R. van de Krol and M. Grätzel, Photoelectrochemical Hydrogen Production, Springer, New York, 2012.

29 R. Memming, Semiconductor Electrolyte, Wiley-VCH, 1999.

30 A. Hagfeldt, U. Björkstén and M. Grätzel, J. Phys. Chem., 1996, 100, 8045-8048.

31 K. Gelderman, L. Lee and S. W. Donne, J. Chem. Educ., 2007, 84, 685-688.

32 L. Ivanova, S. Borisova, H. Eisele, M. Dähne, a. Laubsch and P. Ebert, Appl. Phys. Lett., 2008, 93, 7-10.

33 C. G. Van De Walle and D. Segev, J. Appl. Phys., 2007, 101, 081704.

34 Comsol Multiphysics v5.0, 2015.

35 D. C. Montgomery, Design and Analysis of Experiments, John Wiley \& Sons, Inc., New York, 7th edn, 2008.

36 State-Ease Design Expert v9, 2015.

37 A. Nicolet, S. Guenneau, C. Geuzaine and F. Zolla, J. Comput. Appl. Math., 2004, 168, 321-329.

38 E. A. B. Cole, Mathematical and Numerical Modelling of Heterostructure Semiconductor Devices: From Theory to Programming, Springer, 2009.

39 S. Adachi, Optical Constants of Crystalline and Amorphous Semiconductors: Numerical Data and Graphical Information, Kluwer Academic Publishers, Norwell, MA, 1999.

40 G. M. Hale and M. R. Querry, Appl. Opt., 1973, 12, 555-563.

41 S. Nakamura and M. R. Krames, Proc. IEEE, 2013, 101, 22112220.

42 J. I. Pankove, S. Bloom and G. Harbeke, RCA Rev., 1975, 36, 163.

43 S. Bloom, G. Harbeke, E. Meier and I. B. Ortenburger, Phys. Status Solidi, 1974, 66, 161-168.

44 J. F. Muth, J. H. Lee, I. K. Shmagin, R. M. Kolbas, H. C. Casey, B. P. Keller, U. K. Mishra and S. P. DenBaars, Appl. Phys. Lett., 1997, 71, 2572-2574.
45 J. Piprek, R. Sink, M. A. Hansen, J. E. Bowers and S. P. DenBaars, Proc. SPIE, 2000, 3944, 3912-3944.

46 P. Mackowiak and W. Nakwaski, MRS Internet J. Nitride Semicond. Res., 1998, 3, 1-10.

47 V. Bougrov, M. E. Levinshtein, S. L. Rumyantsev and A. Zubrilov, Properties of Advanced Semiconductor Materials GaN, AlN, InN, BN, SiC, SiGe, John Wiley \& Sons, New York, 2001, vol. 1.

48 T. P. Chow and M. Ghezzo, SiC Power Devices in III-Nitride, SiC and Diamond Materials for Electronic Devices, Material Research Society Symposium Proceedings, Pittsburg, PA, 1996, vol. 423.

49 M. Ono, K. Fujii, T. Ito, Y. Iwaki, A. Hirako, T. Yao and K. Ohkawa, J. Chem. Phys., 2007, 126, 054708.

50 M. Rubin, N. Newman, J. S. Chan, T. C. Fu and J. T. Ross, Appl. Phys. Lett., 1994, 64(1), 64-66.

51 M. Ono, K. Fujii, T. Ito, Y. Iwaki, A. Hirako, T. Yao and K. Ohkawa, J. Chem. Phys., 2007, 126, 054708.

52 A. Nakamura, M. Sugiyama, K. Fujii and Y. Nakano, Jpn. J. Appl. Phys., 2013, 52, 08JN20.

53 O. Madelung, U. Rössler, D. Strauch and S. Adachi, Group IV elements, IV-IV and III-V compounds, Springer, 2001.

54 P. Stallinga, Electrical Characterization of Organic Electronic Materials and Devices, John Wiley \& Sons, Chichester, 2009.

55 J. J. Kelly and R. Memming, J. Electrochem. Soc., 1982, 129, 730-738.

56 Light, Water, Hydrogen, ed. C. A. Grimes, O. K. Varghese and S. Ranjan, Springer, New York, 2008.

57 S. S. Kocha, M. W. Peterson, D. J. Arent, J. M. Redwing, M. A. Tischler and J. A. Turner, J. Electrochem. Soc., 1995, 142, 238-240.

58 J. Y. Kim, J.-W. Jang, D. H. Youn, G. Magesh and J. S. Lee, Adv. Energy Mater., 2014, 4, 1-7.

59 L. Jing, J. Zhou, J. R. Durrant, J. Tang, D. Liu and H. Fu, Energy Environ. Sci., 2012, 5, 6552-6558. 